\title{
THE FAST DECLINING TYPE Ia SUPERNOVA 2003gs, AND EVIDENCE FOR A SIGNIFICANT DISPERSION IN NEAR-INFRARED ABSOLUTE MAGNITUDES OF FAST DECLINERS AT MAXIMUM LIGHT*
}

\author{
Kevin Krisciunas $^{1,2}$, G. H. Marion ${ }^{1,3}$, Nicholas B. SuntzefF ${ }^{1,2}$, Guillaume Blanc ${ }^{4}$, Filomena Bufano ${ }^{5}$, Pablo Candia $^{6}$, \\ Regis Cartier $^{7}$, Nancy Elias-Rosa ${ }^{8}$, Juan Espinoza ${ }^{9}$, David GonZalez ${ }^{9}$, Luis GonZalez ${ }^{10}$, Sergio GonZalez ${ }^{10}$, Samuel \\ D. Gooding ${ }^{1}$, Mario Hamuy ${ }^{7}$, Ethan A. Knox ${ }^{11}$, Peter A. Milne ${ }^{12}$, Nidia Morrell ${ }^{10}$, \\ Mark M. Phillips ${ }^{10}$, Maximilian Stritzinger ${ }^{10}$, and Joanna Thomas-Osip ${ }^{10}$ \\ ${ }^{1}$ Texas A\&M University, Department of Physics and Astronomy, 4242 TAMU, College Station, TX 77843-4242, USA; \\ krisciunas@physics.tamu.edu, suntzeff@physics.tamu.edu,sam.gooding86@gmail.com \\ ${ }^{2}$ George P. and Cynthia Woods Mitchell Institute for Fundamental Physics \& Astronomy, Texas A\&M University, \\ Department of Physics and Astronomy, 4242 TAMU, College Station, TX 77843-4242, USA \\ ${ }^{3}$ University of Texas, Department of Astronomy, Austin, TX 78712, USA; hman@astro.as.utexas.edu \\ ${ }^{4}$ APC, UMR 7164, CNRS, Université Paris 7, CEA, Observatoire de Paris, 11 place Marcelin Berthelot, F-75231, Paris, France; blanc@apc.univ-paris7.fr \\ 5 INAF, Osservatorio Astronomico di Padova, Vicolo dell'Osservatorio 5, I-35122, Padova, Italy; filomena.bufano@ oapd.inaf.it \\ ${ }^{6}$ AURA/Gemini Observatory, Casilla 603, La Serena, Chile; pcandia@ gemini.edu \\ ${ }^{7}$ Universidad de Chile, Departamento de Astronomía, Casilla 36-D, Santiago, Chile; rcartier@ das.uchile.cl, mhamuy@das.uchile.cl \\ ${ }^{8}$ Spitzer Science Center, California Institute of Technology, 1200 E. California Blvd., Pasadena, CA 91125, USA; nelias@ipac.caltech.edu \\ ${ }^{9}$ Cerro Tololo Inter-American Observatory, Casilla 603, La Serena, Chile; juan@ @tio.noao.edu \\ ${ }^{10}$ Las Campanas Observatory, Casilla 601,La Serena, Chile; nmorrell@1co.cl,mmp@1co.cl, mstritzinger@1co.cl, jet@1co.cl \\ ${ }^{11}$ Humboldt State University, 1 Harpst Street, Arcata, CA 95521, USA; eaknox@ gmail.com \\ ${ }^{12}$ University of Arizona, Steward Observatory, 933 N. Cherry Ave., Tucson, AZ 85719, USA; pmilne511@cox.net \\ Received 2009 June 10; accepted 2009 September 19; published 2009 October 27
}

\begin{abstract}
We obtained optical photometry of SN 2003gs on 49 nights, from 2 to 494 days after $T\left(B_{\max }\right)$. We also obtained near-IR photometry on 21 nights. SN 2003gs was the first fast declining Type Ia SN that has been well observed since SN 1999by. While it was subluminous in optical bands compared to more slowly declining Type Ia SNe, it was not subluminous at maximum light in the near-IR bands. There appears to be a bimodal distribution in the near-IR absolute magnitudes of Type Ia SNe at maximum light. Those that peak in the near-IR after $T\left(B_{\max }\right)$ are subluminous in the all bands. Those that peak in the near-IR prior to $T\left(B_{\max }\right)$, such as SN 2003gs, have effectively the same near-IR absolute magnitudes at maximum light regardless of the decline rate $\Delta m_{15}(B)$. Near-IR spectral evidence suggests that opacities in the outer layers of SN 2003gs are reduced much earlier than for normal Type Ia SNe. That may allow $\gamma$ rays that power the luminosity to escape more rapidly and accelerate the decline rate. This conclusion is consistent with the photometric behavior of SN 2003gs in the IR, which indicates a faster than normal decline from approximately normal peak brightness.
\end{abstract}

Key words: supernovae: individual (SN 2003gs) - techniques: photometric

Online-only material: color figures

\section{INTRODUCTION}

Type Ia supernovae ( $\mathrm{SNe}$ ) have been used to obtain accurate cosmological distances, leading to the conclusion that the expansion of the universe is accelerating (Riess et al. 1998; Perlmutter et al. 1999). Several pressing questions regarding the observed properties and the underlying physics of the explosion remain unanswered.

The remarkable homogeneity of Type Ia $\mathrm{SNe}$ has led to general consensus regarding the nature of the progenitor system. Type Ia $\mathrm{SNe}$ are believed to result from the thermonuclear disruption of a carbon-oxygen white dwarf. In the favored scenario, the white dwarf is one component of a close binary, and accretes hydrogen from its companion star. When the white dwarf reaches the Chandrasekhar limit, explosive carbon burning sets in. Burning to nuclear statistical equilibrium ensues, yielding radioactive ${ }^{56} \mathrm{Ni}$.

\footnotetext{
* Based in part on observations taken at the Cerro Tololo Inter-American Observatory, National Optical Astronomy Observatories, which are operated by the Association of Universities for Research in Astronomy, Inc. (AURA) under cooperative agreement with the National Science Foundation. The near-IR photometry from La Silla and Paranal was obtained by the European Supernova Collaboration (ESC).
}

Although the intrinsic peak brightness of Type Ia SNe can vary by up to $2.5 \mathrm{mag}$ in the $B$ band, there exists a tight correlation between the peak luminosity and the shape of the light curve which can be exploited to derive accurate relative distances. The peak brightness is determined by the amount of radioactive ${ }^{56} \mathrm{Ni}$ produced in the explosion. This amount spans $0.09-0.93 M_{\odot}$, although most events cluster in the 0.4-0.7 $M_{\odot}$ range (Stritzinger et al. 2006). The cause of this large variation is not understood. It is becoming increasingly clear that Type Ia SNe are not a one-parameter family (Benetti et al. 2004b).

No two events illustrate this more than the intrinsically subluminous SN 1991bg (Filippenko et al. 1992b; Leibundgut et al. 1993) and the intrinsically overluminous SN 1991T (e.g., Filippenko et al. 1992a), both of which showed photometric and spectroscopic behavior that deviated substantially from normal Type Ia SNe, although other highly peculiar events have been discovered recently, such as SN 2002cx (Li et al. 2003; Branch et al. 2004; Jha et al. 2006).

One way of gaining physical insight is to assemble a sample of Type Ia SNe with complete observational coverage from premaximum to nebular phases, over as wide a wavelength range as possible. Such data can then be used to confront state-ofthe-art explosion and spectral synthesis models. Until recently, 
such data have been non-existent. For the very nearby events $\left(v_{\text {helio }} \lesssim 3000 \mathrm{~km} \mathrm{~s}^{-1}\right)$, this shortcoming resulted in the setting up of the European Supernova Collaboration (ESC) ${ }^{13}$ which has already provided high-quality data for at least half a dozen Type Ia SNe: 2002bo (Benetti et al. 2004a), 2002cv (Elias-Rosa et al. 2008), 2002dj (Pignata et al. 2008), 2002er (Pignata et al. 2004; Kotak et al. 2005), 2003cg (Elias-Rosa et al. 2006), 2003du (Stanishev et al. 2007), 2004dt (Altavilla et al. 2007), 2004eo (Pastorello et al. 2007b), 2005bl (Taubenberger et al. 2008), and 2005cf (Garavini et al. 2007; Pastorello et al. 2007a).

Important observational initiatives have been carried out at Las Campanas Observatory (LCO) and Cerro Tololo InterAmerican Observatory (CTIO) whose goal was to obtain wellsampled optical and near-IR light curves. These endeavors have not been limited to nearby Type Ia SNe.

Since Pskovskii (1977), Phillips (1993), and Hamuy et al. (1995) showed that the absolute magnitudes at maximum light of Type Ia SNe were correlated with their decline rates, ${ }^{14}$ these objects have been subjected to ever more intense scrutiny. Meikle (2000), Krisciunas et al. (2004a), Krisciunas et al. (2004b, 2004c), and Wood-Vasey et al. (2008) have shown that over a wide range of decline rates, the near-IR absolute magnitudes of Type Ia SNe at some epoch with respect to maximum light are essentially constant. In the near-IR, most Type Ia SNe are not just standardizable candles, but very nearly standard candles. The few exceptions are mostly identifiable by their unusual IR light-curve shapes.

Near-IR spectra of SN 2003gs (R. Kotak et al. 2009, in preparation) and SN 1986G (Frogel et al. 1987) reveal the presence of lines from iron group elements that appeared earlier and created stronger features than they do in spectra of normal Type Ia SNe. ${ }^{56} \mathrm{Co}$ is produced by the radioactive decay of ${ }^{56} \mathrm{Ni}$, which is the final burning product produced in the hottest and densest regions of the SN during the explosion. The strong and early presence of iron group elements in SN 2003gs suggests that the outer layers of partially burned ejecta have lower opacities in SN 2003gs than they do in normal Type Ia SNe. This is an important clue to understanding the photometric behavior of SN 2003gs, which indicates a faster than normal decline from approximately normal peak brightness. In SN 2003gs, if the envelope surrounding the iron and cobalt regions is not as deep or more transparent than it is in normal Type Ia SNe, then $\gamma$ rays that power the luminosity will escape more easily and the decline rate will increase.

Here, we present extensive optical (UBVRI) and near-IR $(Y J H K)$ data of the nearby Type Ia SN 2003gs. The $Y$ band is a new photometric band (Hillenbrand et al. 2002) which exploits a relatively clean atmospheric window centered at $\sim 1.035 \mu \mathrm{m}$.

\section{ACQUISITION AND REDUCTION OF PHOTOMETRY}

SN 2003gs was visually discovered by Evans (2003) on 2003 July 29.75 UT. It was located at R.A. = 02:27:38.36, decl. $=-01^{\circ} 09^{\prime} 35^{\prime \prime} .4$ (equinox 2000), 13".4 east, and 14".6 south of the nucleus of the barred spiral galaxy NGC 936. A spectrum obtained with the CTIO $1.5 \mathrm{~m}$ telescope on July 30.4 UT revealed it to be a subluminous Type Ia SN similar to $\mathrm{SNe} 1991 \mathrm{bg}$ and 1999 by at roughly 1 day before maximum

\footnotetext{
13 http://www.mpa-garching.mpg.de/ rtn/

14 The light-curve decline parameter, $\Delta m_{15}(B)$, is defined as the decline in apparent brightness in the first 15 days following $B$-band maximum. The observed range of $\Delta m_{15}(B)$ is $0.81 \pm 0.04$ (SN 1999aa) to $1.93 \pm 0.10$ (SN $1991 \mathrm{bg})$ for the Prieto et al. (2006) templates.
}

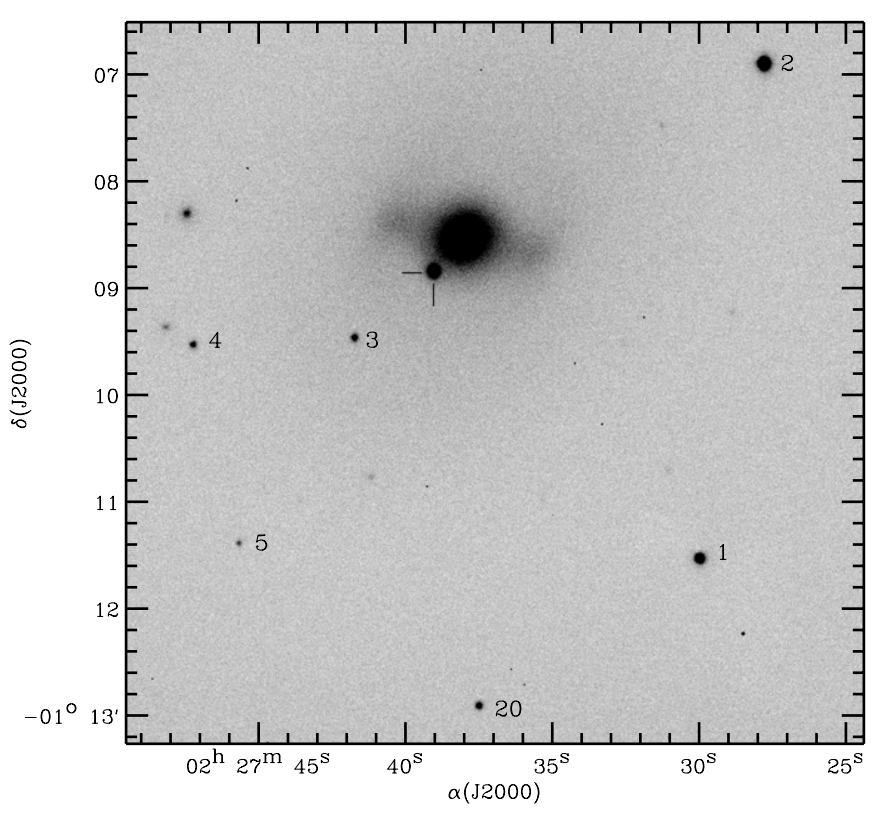

Figure 1. Finder chart for NGC 936, SN 2003gs, and some field stars in our Galaxy. This $6.3 \times 6.3$ arcmin image is a $20 \mathrm{~s} V$-band exposure obtained at 08:56 UT on 2003 August 1 ( $t \sim 4$ days) with the CTIO 1.3 m telescope. North is up and east to the left. The $\mathrm{SN}$ is located 13.4 arcsec east and 14.6 arcsec south of the galaxy core.

Table 1

Properties of SN 2003gs and its Host Galaxy

\begin{tabular}{|c|c|}
\hline Parameter & Value \\
\hline Host galaxy & NGC 936 \\
\hline Host galaxy type $\mathrm{a}^{\mathrm{a}}$ & $\mathrm{SB} 0 / \mathrm{SBa}$ \\
\hline Heliocentric radial velocity ${ }^{\mathrm{a}}$ & $1430 \mathrm{~km} \mathrm{~s}^{-1}$ \\
\hline Distance modulus ${ }^{\mathrm{b}}$ & $31.65 \pm 0.28 \mathrm{mag}$ \\
\hline$E(B-V)_{\mathrm{Gal}}^{\mathrm{c}}$ & $0.035 \pm 0.003 \mathrm{mag}$ \\
\hline R.A. of SN (J2000) & $2^{\mathrm{h}} 27^{\mathrm{m}} 38^{\mathrm{s}} .36$ \\
\hline Decl. of SN (J2000) & $-01^{\circ} 09^{\prime} 35^{\prime \prime} .4$ \\
\hline Offset from nucleus & 13." 4 E 14 ". $6 \mathrm{~S}$ \\
\hline Julian Date of $B$-band maximum & $2452848.80 \pm 0.53$ \\
\hline$\Delta m_{15}(\mathrm{~B})$ & $1.83 \pm 0.02$ \\
\hline$M_{B, \max }$ & $-17.94 \pm 0.29$ \\
\hline$M_{V, \max }$ & $-18.38 \pm 0.29$ \\
\hline$M_{R, \max }$ & $-18.53 \pm 0.29$ \\
\hline$M_{I, \max }$ & $-18.45 \pm 0.29$ \\
\hline$M_{J, \max }$ & $-18.50 \pm 0.29$ \\
\hline$M_{H, \max }$ & $-18.48 \pm 0.29$ \\
\hline$M_{K, \max }$ & $-18.37 \pm 0.29$ \\
\hline
\end{tabular}

Notes.

a From NED.

b Tonry et al. (2001), using method of surface brightness fluctuations, and corrected by 0.16 mag (Jensen et al. 2003).

c Schlegel et al. (1998).

light (Suntzeff et al. 2003; Matheson \& Suntzeff 2003). The spectroscopic typing was confirmed using a spectrum obtained on July 31.33 with the $6.5 \mathrm{~m}$ Baade telescope at LCO (Hamuy et al. 2003). These authors also noted that the spectrum lacked $\mathrm{Na}$ I D lines, suggesting that SN 2003gs is not significantly reddened by dust. Some preliminary photometry was published in the IAU Circulars, including infrared photometry accurate to $\pm 0.1 \mathrm{mag}$ (Mikolajewska \& Szostek 2003), but here we will only consider our final, reduced photometry. Figure 1 shows NGC 936, SN 2003gs, and the local sequence of stars. A list of basic parameters is given in Table 1 . 
Table 2

Optical Field Star Sequence near SN 2003gs

\begin{tabular}{rccccc}
\hline \hline ID & $U$ & $B$ & $V$ & $R$ & $I$ \\
\hline 1 & 16.332 & 15.450 & 14.425 & 13.775 & 13.211 \\
2 & 14.072 & 13.957 & 13.314 & 12.945 & 12.557 \\
3 & $\ldots$ & 17.890 & 16.271 & 15.235 & 13.976 \\
4 & $\ldots$ & 18.125 & 16.575 & 15.520 & 14.147 \\
5 & 18.444 & 18.334 & 17.639 & 17.210 & 16.762 \\
20 & 18.016 & 17.160 & 16.195 & 15.596 & 15.060 \\
\hline
\end{tabular}

SN 2003gs is one of the few fast declining Type Ia SNe to be observed very well both photometrically and spectroscopically at optical and IR wavelengths since SN 1986G (Phillips et al. 1987; Frogel et al. 1987). Three nights of infrared data of the prototypical fast decliner SN 1991bg were published by Krisciunas et al. (2004c). The fast decliner SN 1999by was studied by Garnavich et al. (2004); this object was observed on 14 nights in the infrared, but the IR maxima were not well covered.

The fast decliners SNe 1991bg and 1999by are several tenths of a magnitude fainter in the near-IR than more normal Type Ia SNe. See Figure 16 of Krisciunas et al. (2004c). Given the rarity of fast declining Type Ia SNe, SN 2003gs gives us an opportunity to study an object almost as extreme as these two other examples. ${ }^{15}$

Most of our photometry was obtained with the $1.3 \mathrm{~m}$ telescope at CTIO and the dual optical-infrared imager ANDICAM. The optical channel gives images with a scale of 0.369 arcsec pixel $^{-1}$. The gain is 2.3 electrons per analog-to-digital unit (ADU). The readnoise is 6.5 electrons rms. For the IR channel, the plate scale is 0.137 arcsec pixel $^{-1}$, the gain is 7.2 electrons per ADU, and the readnoise is 20 electrons rms. The optical photometry was derived using point-spread function (PSF) magnitudes.

The optical photometry was calibrated by first determining the UBVRI magnitudes of the field stars near SN 2003gs and tying them to Landolt (1992) standards. One or two Landolt fields were observed along with the SN 2003gs field on nine ostensibly photometric nights using the CTIO $1.3 \mathrm{~m}$. The mean values of the photometry of the secondary standards are given in Table 2. Four nights of optical photometry of the field stars, obtained with the $1 \mathrm{~m}$ Swope telescope at LCO and the CTIO $0.9 \mathrm{~m}$ telescope, confirmed that the BVRI magnitudes of the field stars allowed photometric zero points to be determined to better than \pm 0.02 mag. There may be systematic and random errors in our $U$-band photometry at the $0.08 \mathrm{mag}$ level owing to the non-zero color terms on different systems and the inherently greater scatter of $U$-band photometry.

Optical imagery of SN 2003gs was obtained with the $1 \mathrm{~m}$ Swope telescope at LCO on 16 nights during the Carnegie Type II Supernova (CATS) Survey (Hamuy et al. 2009). Reduction of the photometry from the LCO $1 \mathrm{~m}$ system had to include a term for each filter to account for nonlinearities in the response of the CCD camera (Hamuy et al. 2006).

A further five epochs of optical photometry were obtained with the CTIO $0.9 \mathrm{~m}$ telescope from 2003 August 27 through

\footnotetext{
15 A slowly declining Type Ia SN has decline rate $\Delta m_{15}(B) \lesssim 1.0$. A mid-range decliner has $1.0 \lesssim \Delta m_{15}(B) \lesssim 1.6$. A fast decliner has $\Delta m_{15}(B) \gtrsim$ 1.6. There are spectroscopic differences between the three groups. The slow decliners, for example, show the strongest lines due to doubly ionized species and the weakest lines due to singly ionized species, because they have hotter temperatures.
}

Table 3

Near-Infrared Photometry of Field Star Near SN 2003gs ${ }^{a}$

\begin{tabular}{lcc}
\hline \hline Band & \multicolumn{1}{c}{ Derived $^{\text {b }}$} & 2MASS values \\
\hline$Y$ & $13.221(0.016)$ & $\ldots$ \\
$J$ & $12.779(0.011)$ & $12.812(0.027)$ \\
$H$ & $12.258(0.009)$ & $12.251(0.022)$ \\
$K$ & $11.985(0.014)$ & $11.993(0.019)$ \\
\hline & \\
Notes. & \\
a R.A. = 02:27:40.63, decl. = $-01: 10: 05.1$ (equinox 2000). This is \\
"star 3" of the field star sequence. \\
b With respect to stars 9104 and 9172 of Persson et al. (1998).
\end{tabular}

2004 February 16 UT. The August 27 (CTIO) photometry was reduced using PSF magnitudes. For the final four epochs of CTIO $0.9 \mathrm{~m}$ imagery and the final three epochs of CTIO $1.3 \mathrm{~m}$ imagery, we subtracted template images obtained with the CTIO $0.9 \mathrm{~m}$ on 2007 October 19 UT, long after SN 2003gs had faded.

Some late-time optical photometry was obtained with the University of Arizona $1.54 \mathrm{~m}$ and $2.3 \mathrm{~m}$ telescopes. The results were derived using image subtraction templates obtained in November and December of 2005.

Experiments with the CTIO $0.9 \mathrm{~m}$ imagery with and without image subtraction indicate no statistically significant differences through 57 days after $T\left(B_{\max }\right)$. At $t=91$ days, the $B V R I$ data obtained using image subtraction are on average $0.03 \mathrm{mag}$ fainter than PSF photometry without image subtraction. These differences are comparable to the random errors of the photometry.

We observed two IR standards of Persson et al. (1998), P9104 and P9172, on six photometric nights along with the field of NGC 936 to calibrate the field star immediately southeast of the SN ("star 3" of the photometric sequence). In Table 3, we give the mean $Y J H K$ values of this field star, along with the $J H K$ values from the Two Micron All Sky Survey (2MASS). As one can see, the agreement is good. Since not all the data were taken on photometric nights when IR standards were observed, we derived differential filter-by-filter magnitudes and added these differential values to our derived photometry of the key field star in order to obtain the SN photometry found in Table 5.

We do not rely on measures of $Y$-band standards given by Hillenbrand et al. (2002). Instead, we rely on synthetic photometry of Sirius, Vega, and the Sun. Krisciunas et al. (2004b) give the following relation:

$$
\left(Y-K_{s}\right)=-0.013+1.614\left(J_{s}-K_{s}\right) .
$$

This expression allows us to use the $J_{s}$ and $K_{s}$ magnitudes of Persson et al. (1998) standards to estimate the $Y$-band magnitudes of those standards. The $Y$-band calibrations should only be considered approximate $( \pm 0.03 \mathrm{mag})$.

The UBVRI and YJHK photometry of SN 2003gs is given in Tables 4 and 5 . We note that the $K$-band filter of ANDICAM on the CTIO $1.3 \mathrm{~m}$ is closer to the $K$-short filter used at Las Campanas than a standard, wider $K$-band filter. Throughout this paper what we call $K$-band photometry is really $K_{s}$ photometry.

Figure 2 shows the UBVRI light curves. For the lightcurve fits, we have corrected the ANDICAM $B$ - and $V$-band photometry to the filter system of Bessell (1990). These are the so-called $S$-corrections (Stritzinger et al. 2002; Krisciunas et al. 2003). Without these corrections, the $B-V$ colors of Type Ia $\mathrm{SNe}$ from ANDICAM are systematically too red by as much as $0.1 \mathrm{mag}$. 
Table 4

Optical Photometry of SN 2003gs

\begin{tabular}{|c|c|c|c|c|c|c|c|}
\hline $\mathrm{JD}^{\mathrm{a}}$ & Epoch $^{\mathrm{b}}$ & $U$ & $B$ & $V$ & $R$ & $I$ & Telescope $^{b}$ \\
\hline 2850.80 & +2.0 & $\ldots$ & $14.125(0.016)$ & $13.491(0.014)$ & $13.249(0.015)$ & $13.253(0.014)$ & 2 \\
\hline 2850.91 & +2.1 & $14.731(0.041)$ & $14.134(0.012)$ & $13.488(0.009)$ & $13.279(0.008)$ & $13.387(0.013)$ & 1 \\
\hline 2851.80 & +3.0 & $\ldots$ & $14.235(0.016)$ & $13.511(0.014)$ & $13.263(0.015)$ & $13.244(0.014)$ & 2 \\
\hline 2852.87 & +4.1 & $14.993(0.012)$ & $14.356(0.007)$ & $13.505(0.006)$ & $13.311(0.006)$ & $13.399(0.009)$ & 1 \\
\hline 2854.88 & +6.1 & $15.303(0.021)$ & $14.644(0.013)$ & $13.614(0.011)$ & $13.414(0.003)$ & $13.462(0.010)$ & 1 \\
\hline 2854.88 & +6.1 & $\ldots$ & $14.651(0.018)$ & $13.604(0.015)$ & $13.418(0.012)$ & $13.467(0.014)$ & 1 \\
\hline 2856.86 & +8.1 & $15.761(0.083)$ & $14.982(0.018)$ & $13.781(0.012)$ & $13.544(0.011)$ & $13.503(0.014)$ & 1 \\
\hline 2858.86 & +10.1 & $16.031(0.015)$ & $15.333(0.009)$ & $13.955(0.006)$ & $13.663(0.006)$ & $13.519(0.005)$ & 1 \\
\hline 2861.88 & +13.1 & $16.428(0.015)$ & $15.762(0.009)$ & $14.226(0.006)$ & $13.839(0.006)$ & $13.565(0.010)$ & 1 \\
\hline 2864.86 & +17.1 & $16.587(0.042)$ & $16.047(0.019)$ & $14.515(0.011)$ & $14.080(0.011)$ & $13.699(0.012)$ & 1 \\
\hline 2866.90 & +18.1 & $\ldots$ & $\ldots$ & $14.730(0.015)$ & $\ldots$ & $\ldots$ & 6 \\
\hline 2867.80 & +19.0 & $16.890(0.050)$ & $16.297(0.012)$ & $14.826(0.006)$ & $14.396(0.005)$ & $13.993(0.005)$ & 1 \\
\hline 2870.80 & +22.0 & $\ldots$ & $16.489(0.015)$ & $15.178(0.014)$ & $14.675(0.014)$ & $14.235(0.014)$ & 2 \\
\hline 2872.74 & +23.9 & $17.258(0.144)$ & $16.607(0.020)$ & $15.202(0.013)$ & $14.833(0.012)$ & $14.438(0.015)$ & 1 \\
\hline 2876.80 & +28.0 & $17.274(0.013)$ & $16.718(0.013)$ & $15.394(0.013)$ & $15.061(0.013)$ & $14.715(0.014)$ & 1 \\
\hline 2878.75 & +30.0 & $17.136(0.025)$ & $16.724(0.024)$ & $15.475(0.015)$ & $15.100(0.011)$ & $14.702(0.019)$ & 3 \\
\hline 2882.75 & +34.0 & $17.434(0.051)$ & $16.870(0.016)$ & $15.615(0.011)$ & $15.345(0.011)$ & $15.014(0.014)$ & 1 \\
\hline 2885.73 & +36.9 & $17.505(0.057)$ & $16.912(0.021)$ & $15.730(0.011)$ & $15.469(0.010)$ & $15.138(0.014)$ & 1 \\
\hline 2888.90 & +40.1 & $\ldots$ & $16.957(0.015)$ & $15.904(0.014)$ & $15.575(0.014)$ & $15.252(0.014)$ & 2 \\
\hline 2890.78 & +42.0 & $17.769(0.062)$ & $17.069(0.022)$ & $15.887(0.011)$ & $15.677(0.010)$ & $15.387(0.015)$ & 1 \\
\hline 2890.80 & +42.0 & $\ldots$ & $17.018(0.015)$ & $15.941(0.014)$ & $15.635(0.014)$ & $15.334(0.014)$ & 2 \\
\hline 2897.78 & +49.0 & $18.062(0.088)$ & $17.145(0.021)$ & $16.093(0.016)$ & $15.937(0.014)$ & $15.657(0.015)$ & 1 \\
\hline 2904.73 & +55.9 & $18.345(0.145)$ & $17.281(0.041)$ & $16.333(0.017)$ & $16.192(0.014)$ & $15.958(0.028)$ & 1 \\
\hline 2905.70 & +56.9 & $\ldots$ & $17.244(0.016)$ & $16.376(0.015)$ & $16.181(0.015)$ & $15.957(0.014)$ & 2 \\
\hline 2905.76 & +57.0 & $17.952(0.090)$ & $17.224(0.036)$ & $16.381(0.040)$ & $16.260(0.033)$ & $16.079(0.033)$ & 3 \\
\hline 2906.80 & +58.0 & $\ldots$ & $17.264(0.016)$ & $16.451(0.015)$ & $16.260(0.014)$ & $15.998(0.014)$ & 2 \\
\hline 2907.80 & +59.0 & $\ldots$ & $17.277(0.019)$ & $16.473(0.022)$ & $16.323(0.025)$ & $16.056(0.020)$ & 2 \\
\hline 2908.80 & +60.0 & $\ldots$ & $17.298(0.015)$ & $16.459(0.014)$ & $16.312(0.014)$ & $16.061(0.015)$ & 2 \\
\hline 2911.75 & +63.0 & $18.400(0.039)$ & $17.374(0.015)$ & $16.560(0.011)$ & $16.487(0.011)$ & $16.215(0.012)$ & 1 \\
\hline 2914.80 & +66.0 & $\ldots$ & $17.395(0.019)$ & $16.692(0.016)$ & $16.583(0.014)$ & $16.344(0.016)$ & 2 \\
\hline 2918.72 & +69.9 & $18.470(0.074)$ & $17.502(0.029)$ & $16.767(0.012)$ & $16.759(0.010)$ & $16.457(0.013)$ & 1 \\
\hline 2926.67 & +77.9 & $18.797(0.054)$ & $17.655(0.034)$ & $16.963(0.011)$ & $17.070(0.012)$ & $16.688(0.016)$ & 1 \\
\hline 2939.77 & +91.0 & $\ldots$ & $17.867(0.018)$ & $17.419(0.020)$ & $17.504(0.018)$ & $17.224(0.017)$ & 3 \\
\hline 2952.70 & +103.9 & $\ldots$ & $18.055(0.029)$ & $17.691(0.013)$ & $17.850(0.016)$ & $17.364(0.019)$ & 2 \\
\hline 2967.70 & +118.9 & $\ldots$ & $18.373(0.018)$ & $18.076(0.014)$ & $18.257(0.015)$ & $17.731(0.024)$ & 2 \\
\hline 2996.60 & +147.8 & $\ldots$ & $\ldots$ & $\ldots$ & $\ldots$ & $18.253(0.033)$ & 2 \\
\hline 3000.50 & +151.7 & $\ldots$ & $\ldots$ & $18.712(0.022)$ & $18.941(0.017)$ & $18.361(0.029)$ & 2 \\
\hline 3001.60 & +152.8 & $\ldots$ & $18.934(0.029)$ & $\ldots$ & $\ldots$ & $\ldots$ & 2 \\
\hline 3008.70 & +159.9 & $\ldots$ & $\ldots$ & $19.225(0.035)$ & $19.431(0.058)$ & $18.615(0.061)$ & 4 \\
\hline 3010.70 & +161.9 & $\ldots$ & $19.393(0.076)$ & $19.173(0.065)$ & $19.418(0.025)$ & $18.607(0.027)$ & 4 \\
\hline 3019.60 & +170.8 & $\ldots$ & $19.307(0.038)$ & $19.147(0.019)$ & $19.311(0.022)$ & $18.830(0.040)$ & 2 \\
\hline 3030.57 & +181.8 & $\ldots$ & $19.685(0.036)$ & $19.460(0.047)$ & $19.657(0.038)$ & $19.188(0.041)$ & 3 \\
\hline 3031.56 & +182.8 & $20.548(0.145)$ & $19.975(0.067)$ & $19.474(0.046)$ & $19.724(0.043)$ & $18.868(0.055)$ & 1 \\
\hline 3034.54 & +185.7 & $\ldots$ & $20.143(0.090)$ & $19.432(0.058)$ & $19.946(0.078)$ & $18.968(0.071)$ & 1 \\
\hline 3044.60 & +195.8 & $\ldots$ & $19.665(0.069)$ & $19.893(0.037)$ & $20.107(0.047)$ & $19.228(0.041)$ & 4 \\
\hline 3045.50 & +196.8 & $\ldots$ & 19.767 (0.059) & $19.798(0.037)$ & $20.248(0.105)$ & $19.223(0.043)$ & 4 \\
\hline 3046.60 & +197.8 & $\ldots$ & $19.694(0.054)$ & $19.926(0.039)$ & $20.057(0.061)$ & $19.264(0.083)$ & 4 \\
\hline 3050.52 & +201.7 & $\ldots$ & $20.354(0.138)$ & $19.855(0.044)$ & $20.171(0.083)$ & $19.148(0.088)$ & 1 \\
\hline 3051.55 & +202.7 & $\ldots$ & $19.904(0.405)$ & $19.839(0.126)$ & $20.249(0.267)$ & $19.045(0.144)$ & 3 \\
\hline 3282.90 & +434.1 & $\ldots$ & $22.823(0.293)$ & $22.551(0.473)$ & $\ldots$ & $20.003(0.121)$ & 5 \\
\hline 3283.80 & +435.0 & $\ldots$ & $\ldots$ & $\ldots$ & $22.732(0.344)$ & $\ldots$ & 5 \\
\hline 3284.80 & +436.0 & $\ldots$ & $22.516(0.295)$ & $22.789(0.666)$ & $\ldots$ & $\ldots$ & 5 \\
\hline 3342.80 & +494.0 & $\ldots$ & $\ldots$ & $22.911(0.350)$ & $\ldots$ & $\ldots$ & 5 \\
\hline
\end{tabular}

\section{Notes.}

a Julian Date minus 2,450,000.

b Days since $T\left(B_{\max }\right)$.

c Telescope: 1, CTIO 1.3 m; 2, Las Campanas 1.0 m; 3, CTIO 0.9 m; 4, Univ. Arizona 1.54 m; 5, Univ. Arizona 2.3 m; 6 , Magellan No. 2 (Clay) telescope.

To determine the time of maximum light and the decline rate, we used the light-curve analysis method of Prieto et al. (2006). This method relies on the well-sampled light curves of 14 objects. The two fastest decliners in the training set are $\mathrm{SNe} 1992 \mathrm{bo}$ and $1991 \mathrm{bg}$, which have $\Delta m_{15}(B)=1.69$ and
1.93 , respectively. We find that $\mathrm{SN} 2003 \mathrm{gs}$ had a decline rate of $\Delta m_{15}(B)=1.83 \pm 0.02$. The time of $B$-band maximum was JD $2452848.80 \pm 0.53$. In Figure 2, we show the BVRI templates corresponding to $\Delta m_{15}(B)=1.83$ and adjusted in the $Y$-direction to give the best fits. Figure 3 shows the residuals of 
Table 5

Near-Infrared Photometry of SN 2003gs

\begin{tabular}{|c|c|c|c|c|c|c|c|}
\hline UT date & $\mathrm{JD}^{\mathrm{a}}$ & Epoch $^{\text {b }}$ & $Y$ & $J$ & $H$ & $K$ & Telescope $^{\mathrm{b}}$ \\
\hline Jul 30 & 2850.91 & +2.1 & $13.44(0.02)$ & $13.41(0.01)$ & $13.38(0.01)$ & $13.40(0.02)$ & 1 \\
\hline Aug 1 & 2852.85 & +4.1 & $\ldots$ & $13.55(0.01)$ & $13.45(0.01)$ & $13.38(0.02)$ & 2 \\
\hline Aug 1 & 2852.89 & +4.1 & $13.51(0.02)$ & $13.57(0.02)$ & $13.43(0.02)$ & $13.47(0.02)$ & 1 \\
\hline Aug 3 & 2854.86 & +6.1 & $13.52(0.02)$ & $13.81(0.02)$ & $13.43(0.01)$ & $13.48(0.02)$ & 1 \\
\hline Aug 5 & 2856.79 & +8.0 & $\ldots$ & $14.01(0.02)$ & $13.47(0.01)$ & $13.43(0.02)$ & 2 \\
\hline Aug 5 & 2856.88 & +8.1 & $13.53(0.02)$ & $13.95(0.02)$ & $13.46(0.02)$ & $\ldots$ & 1 \\
\hline Aug 7 & 2858.87 & +10.1 & $13.47(0.02)$ & $14.07(0.02)$ & $13.48(0.01)$ & $13.46(0.02)$ & 1 \\
\hline Aug 10 & 2861.90 & +13.1 & $13.38(0.02)$ & $14.13(0.02)$ & $13.54(0.01)$ & $13.55(0.02)$ & 1 \\
\hline Aug 11 & 2863.79 & +15.0 & $\ldots$ & $14.07(0.02)$ & $13.67(0.01)$ & $13.55(0.02)$ & 2 \\
\hline Aug 13 & 2864.88 & +16.1 & $13.32(0.02)$ & $14.09(0.03)$ & $13.65(0.02)$ & $13.68(0.02)$ & 1 \\
\hline Aug 16 & 2867.81 & +19.0 & $13.43(0.02)$ & $14.30(0.02)$ & $13.90(0.02)$ & $13.94(0.03)$ & 1 \\
\hline Aug 20 & 2871.80 & +23.0 & $\ldots$ & $14.79(0.03)$ & $14.32(0.01)$ & $14.37(0.02)$ & 2 \\
\hline Aug 21 & 2872.76 & +24.0 & $13.91(0.03)$ & $15.06(0.04)$ & $14.40(0.04)$ & $14.49(0.09)$ & 1 \\
\hline Aug 25 & 2876.83 & +28.0 & $14.10(0.02)$ & $15.44(0.03)$ & $14.59(0.03)$ & $14.77(0.04)$ & 1 \\
\hline Aug 31 & 2882.77 & +34.0 & $14.51(0.03)$ & $15.96(0.07)$ & $15.02(0.04)$ & $15.01(0.08)$ & 1 \\
\hline Sep 6 & 2888.88 & +40.1 & $\ldots$ & $16.55(0.04)$ & $15.31(0.03)$ & $15.47(0.03)$ & 2 \\
\hline Sep 8 & 2890.77 & +42.0 & $14.93(0.03)$ & $16.79(0.12)$ & $15.25(0.05)$ & $15.54(0.06)$ & 1 \\
\hline Sep 15 & 2897.80 & +49.0 & $\ldots$ & $16.98(0.11)$ & $15.41(0.05)$ & $\ldots$ & 1 \\
\hline Sep 18 & 2900.78 & +52.0 & $\ldots$ & $17.36(0.05)$ & $15.86(0.06)$ & $16.13(0.05)$ & 2 \\
\hline Sep 29 & 2911.75 & +63.0 & $\ldots$ & $17.69(0.22)$ & $15.94(0.07)$ & $\ldots$ & 1 \\
\hline Oct 6 & 2918.74 & +70.0 & $\ldots$ & $18.12(0.20)$ & $16.12(0.09)$ & $\ldots$ & 1 \\
\hline Oct 14 & 2926.69 & +77.9 & $\ldots$ & $18.51(0.28)$ & $16.60(0.08)$ & $\ldots$ & 1 \\
\hline 2004 Aug 3 & 3220.82 & +372.0 & $\ldots$ & $>21.50$ & $>20.60$ & $>20.80$ & 3 \\
\hline
\end{tabular}

Notes.

a Julian Date minus 2,450,000.

b 1, CTIO $1.3 \mathrm{~m}$ + ANDICAM. 2, ESO $3.6 \mathrm{~m} \mathrm{NTT}+$ SofI. $3=$ VLT + ISAAC.

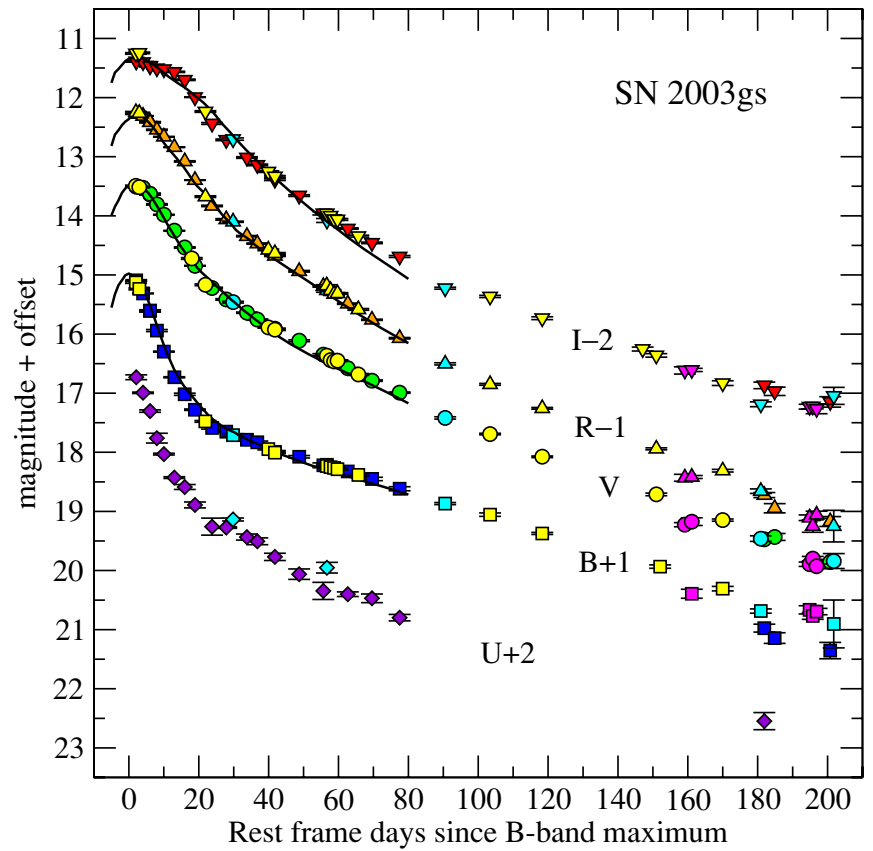

Figure 2. Optical light curves of SN 2003gs. Yellow symbols represent data obtained at Las Campanas. Cyan symbols represent data obtained with the CTIO $0.9 \mathrm{~m}$. Magenta symbols represent data obtained with the University of Arizona $1.54 \mathrm{~m}$ telescope. All other data were obtained with the CTIO $1.3 \mathrm{~m}$ telescope. The data have been shifted vertically for clarity by the amounts indicated. The solid lines represent the $\Delta m_{15}(B)=1.83$ set of light-curve templates of Prieto et al. (2006), adjusted to minimize the total $\chi^{2}$ of the fits.

(A color version of this figure is available in the online journal.)

the photometry with respect to the family of $\Delta m_{15}(B)=1.83$ templates. The I-band data, in particular, did not correspond well to any template of Prieto et al. (2006). We note with satisfaction, however, that data from different telescopes are in reasonable

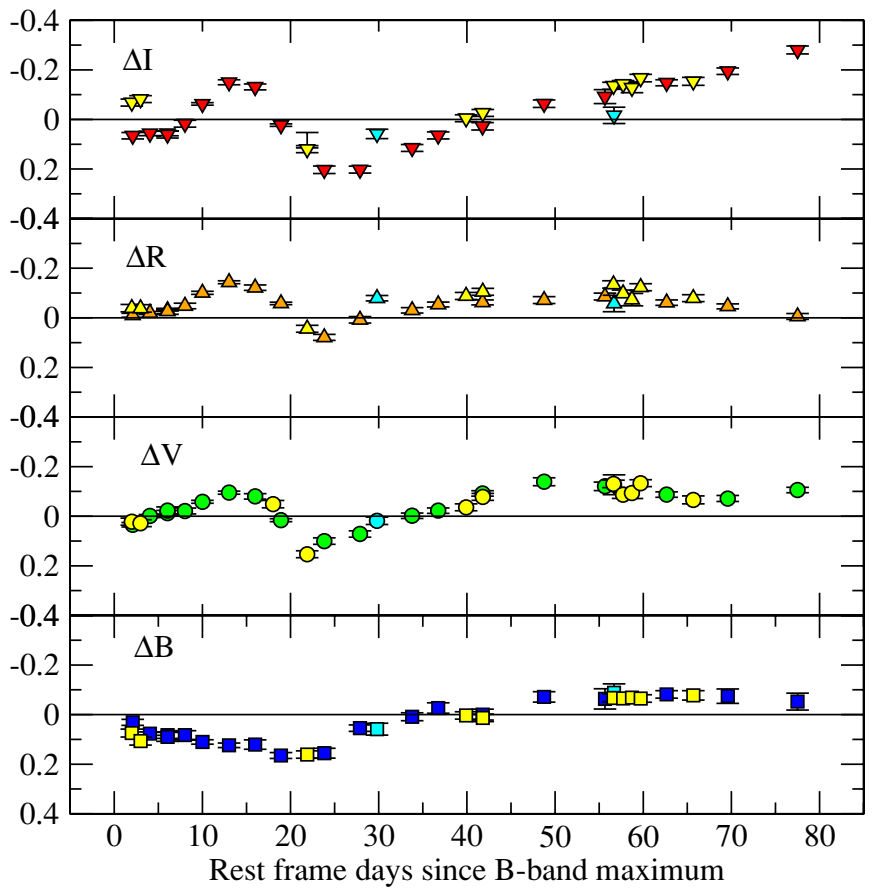

Figure 3. Residuals of $B V R I$ photometry with respect to $\Delta m_{15}(B)=1.83$ templates shown in Figure 2.

(A color version of this figure is available in the online journal.)

agreement with each other, better in fact than the agreement of the data with the family of $\Delta m_{15}(B)=1.83$ templates.

Figure 4 shows the YJHK light curves. The CTIO $1.3 \mathrm{~m}$ photometry includes the $S$-corrections given in Table 6. Given that we had access to the IR spectra of SN 2003gs, it was possible to place the CTIO $1.3 \mathrm{~m}$ IR photometry on the photometric 


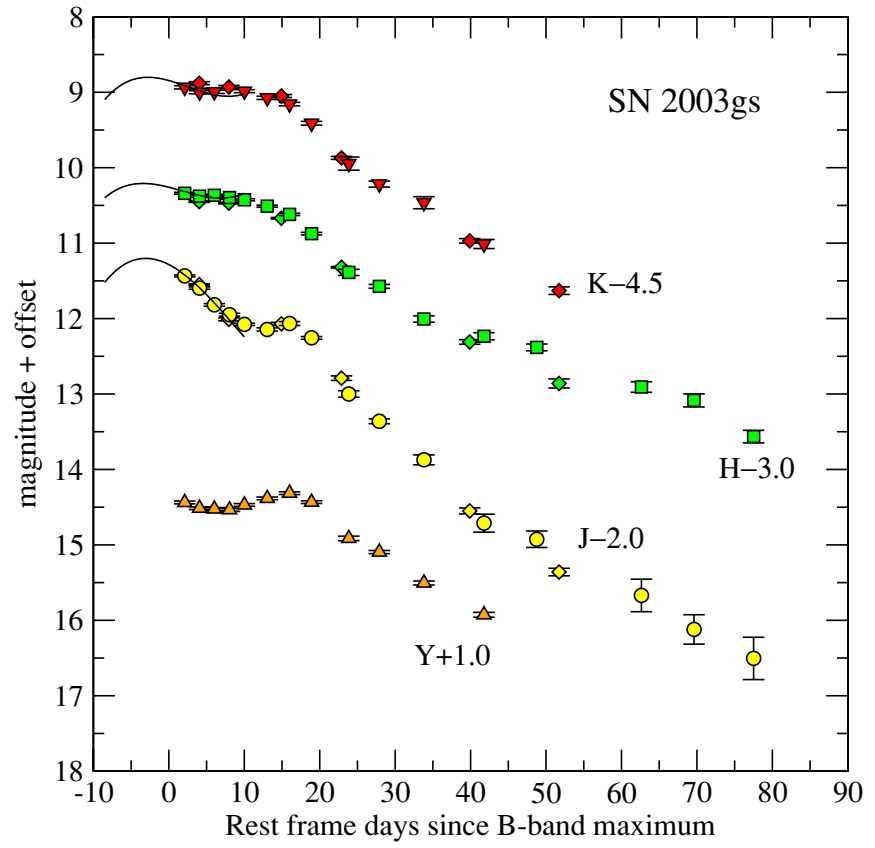

Figure 4. Near-IR photometry. The diamond-shaped symbols represent data obtained with the New Technology Telescope. The other data were taken with the CTIO $1.3 \mathrm{~m}$. The data have been offset vertically for clarity by the number of magnitudes given. We have added the $J H K$ maximum-light templates of Krisciunas et al. (2004b).

(A color version of this figure is available in the online journal.)

Table 6

$S$-Corrections for CTIO 1.3 m Near-IR Photometry ${ }^{a}$

\begin{tabular}{cccc}
\hline \hline$t$ & $\Delta J$ & $\Delta H$ & $\Delta K$ \\
\hline 2.1 & {$[0.028]$} & {$[-0.040]$} & {$[0.031]$} \\
4.1 & 0.028 & -0.053 & 0.031 \\
6.1 & 0.009 & -0.063 & 0.019 \\
8.1 & 0.000 & -0.064 & $\ldots$ \\
10.1 & 0.004 & -0.050 & 0.022 \\
13.1 & 0.011 & -0.027 & 0.027 \\
16.1 & -0.027 & -0.026 & -0.019 \\
19.0 & -0.043 & -0.022 & -0.032 \\
24.0 & -0.063 & -0.014 & -0.045 \\
28.0 & -0.081 & -0.012 & -0.057 \\
34.0 & -0.082 & -0.014 & -0.050 \\
42.0 & -0.076 & -0.019 & -0.034 \\
49.0 & -0.059 & -0.025 & $\ldots$ \\
63.0 & -0.020 & -0.037 & $\ldots$ \\
70.0 & {$[0.000]$} & {$[-0.037]$} & $\ldots$ \\
77.9 & {$[0.000]$} & {$[-0.037]$} & $\ldots$
\end{tabular}

Note.

a These values are to be added to the values in Table 5 to place the ANDICAM near-IR photometry on the photometric system of Persson et al. (1998). $t$ is the number of days since $B$-band maximum.

system of Persson et al. (1998) using the algorithm of Stritzinger et al. (2002) and Krisciunas et al. (2003).

According to the light-curve fits, the first observations of SN 2003gs were obtained on the date of the $V$-band maximum, some 2 days after $T\left(B_{\max }\right)$. We can say with certainty that the times of maximum light of SN 2003gs in the IJHK bands did not occur several days after $T\left(B_{\max }\right)$. The timing of the IR maxima may be related to the range of near-IR absolute magnitudes at maximum light (see Section 3.1 below).

Figure 5 shows the $B-V$ colors of SN 2003gs, the zero reddening line of Lira (1995), and the $B-V$ color excess.

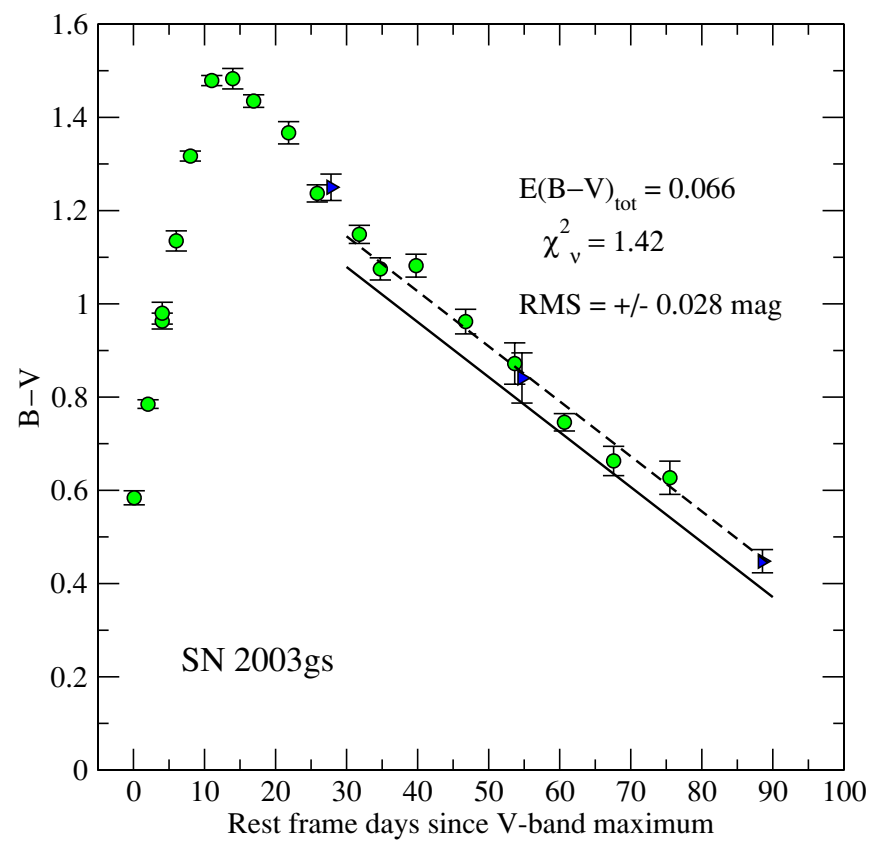

Figure 5. $B-V$ colors of $\mathrm{SN} 2003 \mathrm{gs}$ as a function of time since $V$-band maximum. The right pointing triangles are CTIO $0.9 \mathrm{~m}$ data. The other data are from the CTIO $1.3 \mathrm{~m}$, with the $S$-corrections applied. The solid line is the locus of Lira (1995) for unreddened Type Ia SNe. The dashed line represents an offset corresponding to $E(B-V)=0.066 \mathrm{mag}$.

(A color version of this figure is available in the online journal.)

We note that the Lira line was derived from observations made with the CTIO $0.9 \mathrm{~m}$. We derive statistically equivalent values of $\mathrm{E}(B-V)$ using the two nights of CTIO 0.9 m photometry, or using them in combination with eight nights of corrected ANDICAM photometry, implying that the $S$-corrections were reasonably appropriate. Without adding the $S$-corrections to the ANDICAM $B$ - and $V$-band photometry, our value of $E(B-V)$ would be systematically too red by about 0.1 mag (see Krisciunas et al. 2003, Figure 10). The total $V$-band extinction would then be systematically too large by $\sim 0.3 \mathrm{mag}$, given standard dust (Cardelli et al. 1989).

We find $E(B-V)_{\text {tot }}=0.066 \mathrm{mag}$, of which $0.035 \mathrm{mag}$ is due to dust in our Galaxy (Schlegel et al. 1998). Thus, SN 2003gs is almost unreddened in its host. Assuming $R_{V}=3.1$, we obtain total extinction corrections of $0.270,0.205,0.170,0.124,0.058$, 0.037 , and $0.024 \mathrm{mag}$ for the BVRIJHK bands, respectively, using the scale factors given by Krisciunas et al. (2006, Table 8). As we have found before, the uncertainties in the near-IR extinction corrections are comparable to the random errors of the photometry, even if a given object is dimmed and reddened by dust with non-standard properties.

In Table 1, we give the absolute magnitudes of SN 2003gs for the $B V R I$ and $J H K$ bands. These rely on the distance modulus of NGC 936 of $m-M=31.81 \pm 0.28$ mag given by Tonry et al. (2001), based on the method of surface brightness fluctuations (SBFs), and corrected by $0.16 \mathrm{mag}$ to $m-M=31.65 \mathrm{mag}$ to account for a systematic error in the $I$-band Cepheid periodluminosity relation used for the calibration of the SBF distances (Jensen et al. 2003). We have adopted the total extinction values given above.

Garnavich et al. (2004, Equations (2)-(4)) give exponential fits to the $B V I$ decline rate relations, which fit the absolute magnitudes over the full range of decline rates of Type Ia SNe. While SN 2003gs was subluminous compared to mid-range 
Table 7

Low-Order Polynomial Fits to $V-H$ and $V-K$ Colors $^{\mathrm{a}}$

\begin{tabular}{lcccc}
\hline \hline Color Index & $V-H$ & $V-H$ & $V-K$ & $V-K$ \\
\hline Range (days) & {$[-9,+16.5]$} & {$[+18.5,+88.5]$} & {$[-8,+16]$} & -0.152 \\
$\mathrm{a}_{0}$ & 0.091 & 1.399 & $-0.2787401 \mathrm{E}-02$ & 1.671 \\
$\mathrm{a}_{1}$ & $-0.5126819 \mathrm{E}-02$ & $-0.3641677 \mathrm{E}-01$ & $-0.5979778 \mathrm{E}-01$ \\
$\mathrm{a}_{2}$ & $+0.3556262 \mathrm{E}-02$ & $+0.3332700 \mathrm{E}-03$ & $+0.7684491 \mathrm{E}-02$ & $+0.7580789 \mathrm{E}-03$ \\
$\mathrm{a}_{3}$ & $-0.1658202 \mathrm{E}-04$ & $-0.1711514 \mathrm{E}-05$ & $-0.2259151 \mathrm{E}-03$ & $-0.4665809 \mathrm{E}-05$ \\
rms (mag) & \pm 0.172 & \pm 0.101 & \pm 0.209 & \pm 0.107 \\
$\chi_{v}^{2}$ & 23.9 & 2.1 & 15.5 & 2.9 \\
\hline
\end{tabular}

Note.

${ }^{\text {a }}$ Fits are of the form: $V-H$ or $V-K=a_{0}+\Sigma\left(a_{i} t^{i}\right)$.

decliners at optical wavelengths, we find that SN 2003gs was $0.18,0.15$, and 0.30 mag brighter in the $B, V$, and $I$ bands, respectively, than the values implied by the relationships of Garnavich et al. (2004) for a Type Ia SN with $\Delta m_{15}(B)=1.83$.

The $J$-band data just after maximum light are convincingly fit with the unstretched template given by Krisciunas et al. (2004b). The implication is that the $J$-band maximum was $0.23 \mathrm{mag}$ brighter than our first ( $S$-corrected) value, obtained 2.1 days after $T\left(B_{\max }\right)$. That the unstretched template fits the data is evidence that in the $J$ band the photometric behavior of SN 2003gs was more like a mid-range decliner than a fast decliner. If we use the unstretched maximum light $H$ - and $K$-band templates of Krisciunas et al. (2004b), the $H$ - and $K$-band maxima were approximately 0.13 mag brighter than our earliest observations. Along with the small near-IR extinctions and a distance modulus of $31.65 \mathrm{mag}$, the resulting absolute magnitudes are $M_{J}=$ $-18.50, M_{H}=-18.48$, and $M_{K}=-18.37$. These values are $0.11 \mathrm{mag}$ fainter in $M_{J}, 0.18 \mathrm{mag}$ brighter in $M_{H}$, and $0.07 \mathrm{mag}$ fainter in $M_{K}$ than the mean values of the slow decliners and mid-range decliners (see below).

In Figure 6, we show the unreddened $V$ minus near-IR colors of SN 2003gs and several other Type Ia SNe: the very normal mid-range decliner 2001el (Krisciunas et al. 2003); and the fast decliners 1999by (Garnavich et al. 2004), 2005bl (Taubenberger et al. 2008; Wood-Vasey et al. 2008), 2005ke, and $2006 \mathrm{mr}$ (Contreras et al. 2009; Folatelli et al. 2009). We have also used the $K$-band data of SN 2005ke from Wood-Vasey et al. (2008). SNe 199by, 2003gs, 2005bl, and 2005ke show similar $V-H$ and $V-K$ colors from the earliest times to $t \sim+16$ days. There is a ridge line onto which $V-H$ and $V-K$ colors converge, starting at $t \sim+18.5$ days for the fast decliners; these loci meet up with the SN 2001el data starting at $t \sim 30$ days.

In Table 7, we give low-order polynomial fits to the earlyand late-time $V-H$ and $V-K$ colors shown in Figure 6. The early-time colors of SNe 2001el and 2006mr have been excluded from the fits. Given the reduced $\chi^{2}$ values and the $\pm 0.2 \mathrm{mag}$ rms scatter of the fits to the early-time data of SNe 1999by, 2003gs, $2005 \mathrm{bl}$, and 2005ke, we should not consider these colors to be "uniform." But we find that the $V-H$ and $V-K$ colors of the fast decliners are remarkably uniform after $t \sim+30$ days, and identical to the unreddened colors of the mid-range decliner SN 2001el. The rms scatter in the two color indices for the linear decline is $\pm 0.1 \mathrm{mag}$. We are reminded of the so-called "Lira law" for the $B-V$ colors of Type Ia SNe starting at $t \sim$ +32 days. The only late-time outliers in the $V-H$ and $V-K$ plots are the $V-H$ data of SN 2003gs, which are apparently redder than other objects due to an interesting bump in the $\mathrm{H}$ band spectra of SN 2003gs after $t \sim+45$ days (R. Kotak et al. 2009 , in preparation).

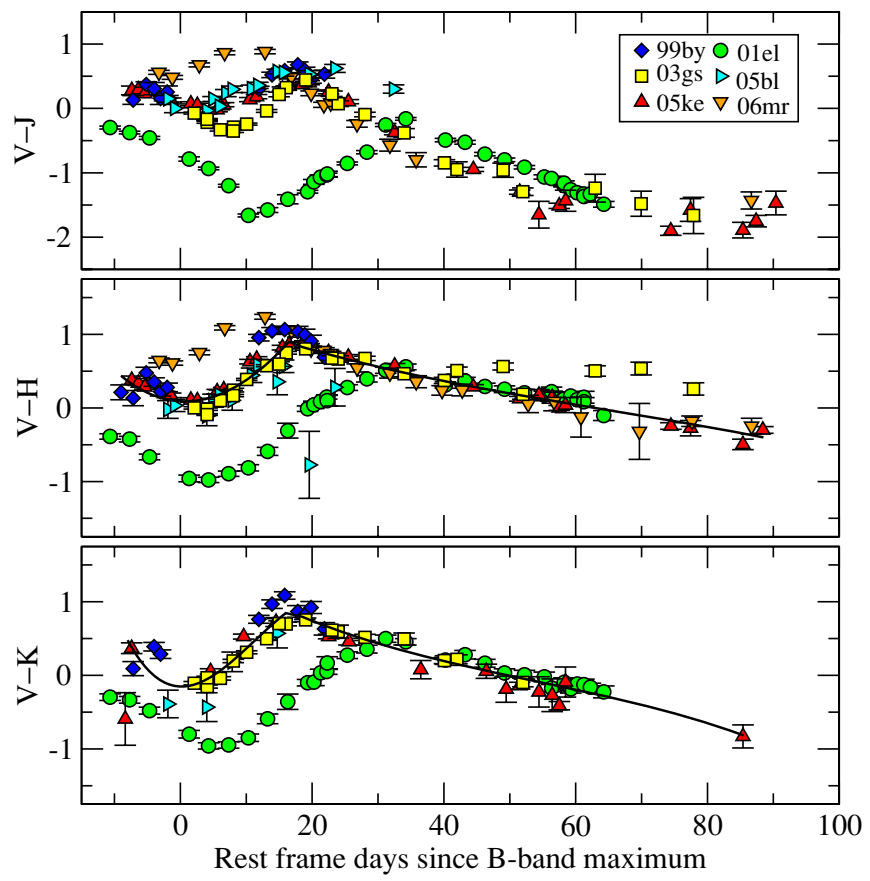

Figure 6. $V-J, V-H$, and $V-K$ colors of Type Ia SNe. The photometry has been corrected for Galactic reddening and host galaxy reddening. The low-order polynomial fits to subsets of the $V-H$ and $V-K$ data given in Table 7 are also shown here. Prior to $t=17$ days, the fits have a large reduced $\chi^{2}$, but after that the nearly linear fits are considerably tighter and even match the colors of the normal Type Ia SN 2001el.

(A color version of this figure is available in the online journal.)

\section{DISCUSSION}

\subsection{Photometry}

\subsubsection{Light-Curve Morphology}

Hamuy et al. (1996) first showed that a stack of $I$-band light curves, ordered by the optical decline rate parameter, exhibits weaker and weaker secondary maxima as we proceed from the slowest to the fastest decliners. If we consider the mean flux 20 40 days after the time of $B$-band maximum, typical mid-range decliners have a secondary maximum that is 0.5 times as strong as the $I$-band maximum (Krisciunas et al. 2001, Figure 17). For the fast decliners, the second flux peak is weak enough that it just blends in with the principal decline.

SN 2003gs exhibited no secondary hump in the $I, H$, and $K$ bands. There is only a weak $J$-band secondary hump. In the $Y$ band, however, SN 2003gs had a secondary maximum that was brighter than the SN must have been at the time of $B$-band maximum. This was also the case with the mid-range 


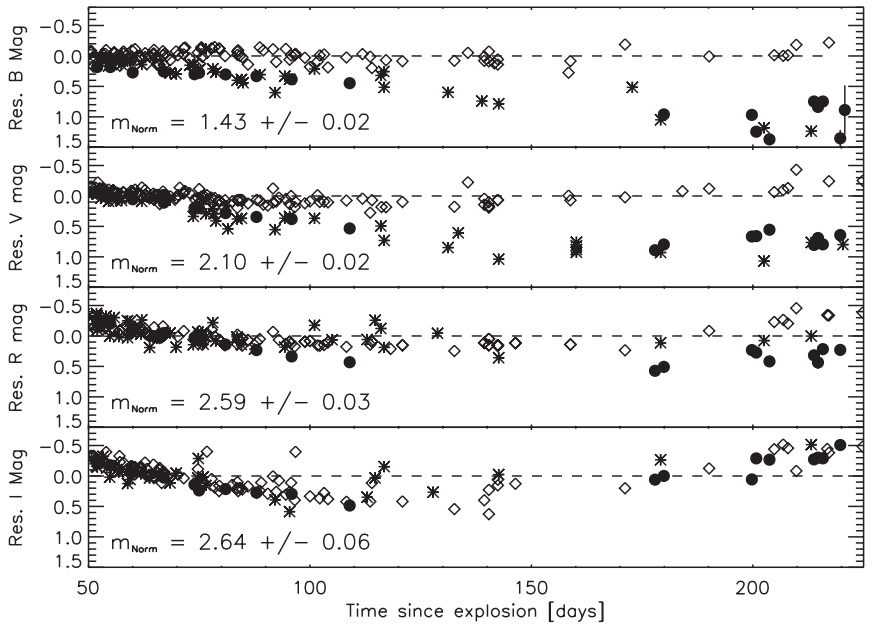

Figure 7. Residual photometric brightness with respect to normal Type Ia supernovae (dashed line). From 50 to 200 days after the time of explosion, Type Ia SNe light curves exhibit a reasonably linear decline. Here, we plot the differences of the photometry with respect to the BVRI rates of decline of normal objects. Symbols: diamonds, normal Type Ia SNe; dots, SN 2003gs; asterisks, other fast decliners.

decliner SN 2000bh (Krisciunas et al. 2004b). Many Type Ia SNe observed by the Carnegie Supernova Project ${ }^{16}$ (CSP) show the same phenomenon (Contreras et al. 2009). The secondary hump is apparently maximized in the $1.03 \mu \mathrm{m}$ band. According to Kasen (2006), it is due to a change of opacity, when the expanding fireball undergoes a transition from primarily doubly ionized species to singly ionized ones.

Milne et al. (2001) studied the late-time light-curve evolution of Type Ia SNe. From 50 to 200 days after the time of explosion (i.e., about 30-180 days after the time of $B$-band maximum) normal Type Ia SNe exhibit linearly declining light curves, with rates of decline of $1.43,2.12,2.62$, and 2.67 mag per 100 days in the $B V R I$ bands, respectively. In Figure 7, we show the differences of the photometry with respect to these nominal rates of decline. The 50-200 day rates of decline for SN $2003 \mathrm{gs}$ are similar, but not exact matches, to the other six subluminous objects. The $B$ - and $V$-band rates of decline for the fast declining objects differ appreciably from the normal events, but for the $R$ and $I$ bands there is less variation.

Two excellent reviews of the photometric and spectroscopic properties of fast declining Type Ia SNe have been published by Garnavich et al. (2004) and Taubenberger et al. (2008). Of greatest interest to us here is the intrinsic brightness of SN 2003gs and other fast decliners.

\subsubsection{Fast Decliners to Consider}

Krisciunas et al. (2004a) and Krisciunas et al. (2004c) have already considered the absolute peak magnitudes in the nearIR of SNe 1986G (Frogel et al. 1987; Phillips et al. 1987), 1991bg (Filippenko et al. 1992b; Leibundgut et al. 1993), and 1999by (Garnavich et al. 2004). Here, we use a corrected distance modulus to NGC 5128 (the host of SN 1986G) of $m-M=27.90 \pm 0.14$ mag by subtracting 0.16 mag (Jensen et al. 2003) from the SBF distance modulus of Ajhar et al. (2001, Table 3). This is related to a systematic error in the calibration of the I-band Cepheid period-luminosity relation used to anchor the SBF distances. Like SN 2003gs, SN 1986G appears to be a fast decliner that did not peak "late" in the near-IR. One concern is that this object suffered significant extinction. However, from

\footnotetext{
16 http://csp1.lco.cl/ cspuser1/PUB/CSP.html
}

polarimetry data we know that $R_{V}=2.4$ is the appropriate value to use for dust in the host of SN 1986G (Hough et al. 1987). As with many objects, a larger source of uncertainty in the near-IR absolute magnitudes comes from the uncertainty of the distance modulus, not the uncertainty in the near-IR extinction corrections.

Other fast decliners we can consider are these:

SN 2003hv. Leloudas et al. (2009) present spectra and extensive photometry. It had a decline rate of $\Delta m_{15}(B)=1.61$ \pm 0.02 . The distance modulus is $m-M=31.53 \pm 0.30 \mathrm{mag}$ (Tonry et al. 2001), which becomes $m-M=31.37$ mag after applying the correction of Jensen et al. (2003). This object was unreddened in its host. We estimate that SN 2003hv peaked in the $J$ band 1.7 days prior to $T\left(B_{\max }\right)$. The absolute magnitudes at maximum were $M_{J} \approx-18.52, M_{H} \approx-18.17$, and $M_{K} \approx-18.33$, with uncertainties of \pm 0.31 mag. The peak brightness and the application of Arnett's Law (Arnett 1982) suggest that it produced $0.40-0.42 M_{\odot}$ of ${ }^{56} \mathrm{Ni}$, somewhat more than was produced by the fastest decliners (see Table 9).

SN 2004gs (Contreras et al. 2009; Folatelli et al. 2009). $\Delta m_{15}(B)=1.54 \pm 0.01$. This was a reasonably fast decliner, but the first IR data were obtained at +6.6 days after $T\left(B_{\max }\right)$. We do not know if it peaked early or late, and to extrapolate back to the IR maxima assumes that its light curve obeyed templates based on other objects.

SN 2005bl (Taubenberger et al. 2008; Folatelli et al. 2009; Wood-Vasey et al. 2008). We adopt $\Delta m_{15}(B)=1.80 \pm 0.04$ (Folatelli et al. 2009). Taubenberger et al. (2008) give $\Delta m_{15}(B)$ $=1.93 \pm 0.10$. The $I J H K$ maxima occurred a few days after $T\left(B_{\max }\right)$. The distance modulus is $m-M=35.10 \pm$ 0.09 mag, given the radial velocity in the frame of the cosmic microwave background, $v_{\mathrm{CMB}}=7534 \mathrm{~km} \mathrm{~s}^{-1} \mathrm{Mpc}^{-1}$, and a Hubble constant of $72 \mathrm{~km} \mathrm{~s}^{-1} \mathrm{Mpc}^{-1}$ (Freedman et al. 2001). $E(B-V)_{\text {tot }} \approx 0.20 \pm 0.08$, giving $J H K$ extinctions of 0.17 , 0.11 , and $0.07 \mathrm{mag}$, respectively, with uncertainties of $40 \%$. Using the $J H K$ apparent magnitudes at maximum of WoodVasey et al. (2008), these extinctions, and the Hubble flow distance modulus, we obtain absolute magnitudes at maximum light of $M_{J}=-17.92 \pm 0.12, M_{H}=-17.88 \pm 0.12$, and $M_{K}=-17.70 \pm 0.17$.

SN 2005ke (Contreras et al. 2009; Folatelli et al. 2009). $\Delta m_{15}(B)=1.76 \pm 0.01$. The near-IR maxima occurred 1-2 days after $T\left(B_{\max }\right)$. Its host was NGC 1371, a member of the Eridanus group. Tonry et al. (2001, Table 4) give an SBF distance modulus of $m-M=32.00 \pm 0.08$ mag for the group, which becomes $m-M=31.84$ mag after the Jensen et al. (2003) correction. Using the zero reddening line of Lira (1995), we find $E(B-V)_{\text {tot }}=0.066$, giving $J H K$ extinctions of $0.058,0.037$, and $0.024 \mathrm{mag}$. The Las Campanas photometry gives $J_{\max }=14.00 \pm$ 0.02 and $H_{\max }=13.95 \pm 0.03$. We adopt $K_{\max }=14.03 \pm 0.02$ (Wood-Vasey et al. 2008). The resulting absolute magnitudes are $M_{J}=-17.90, M_{H}=-17.93$, and $M_{K}=-17.83$, to which we assign conservative uncertainties of \pm 0.24 mag. We note that SN 2005ke showed evidence of interacting with the nearby circumstellar medium, based on X-ray observations with Swift (Immler et al. 2006).

SN 2006gt (Contreras et al. 2009; Folatelli et al. 2009). $\Delta m_{15}(B)=1.66 \pm 0.03$. This was a distant fast decliner (redshift $=0.0448)$. The $J$-band maximum clearly occurred prior to $T\left(B_{\max }\right)$. If we simply take the earliest available IR observations and apply no extinction corrections at all, we get $M_{J}=-18.45$ and $M_{H}=-18.21$. This appears to be another object that peaked early and was not faint. 


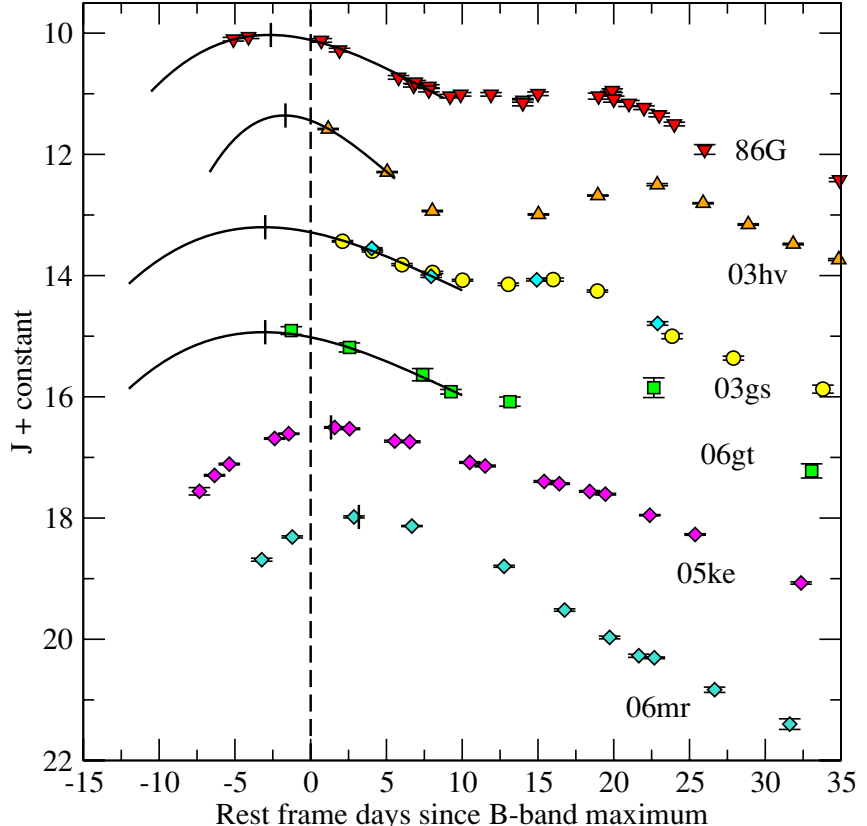

Figure 8. $J$-band photometry of six fast declining Type Ia SNe. The data of SN 2003hv have been offset by $-1.5 \mathrm{mag} ; 2006 \mathrm{gt}$ by $-3.0 \mathrm{mag}$; 2005ke by $+2.5 \mathrm{mag}$; and $2006 \mathrm{mr}$ by $+4.0 \mathrm{mag}$. The peaks of the top four objects are fitted with the stretchable template of Krisciunas et al. (2004b). The stretch factors were 0.877 for SN $1986 \mathrm{G}, 0.556$ for $2003 \mathrm{hv}, 1.00$ for $2003 \mathrm{gs}$, and 1.00 for $2006 \mathrm{gt}$. The top four objects peaked prior to $T\left(B_{\max }\right)$, while the bottom two peaked after $T\left(B_{\max }\right)$. Note also the weak secondary maxima in the bottom two objects.

(A color version of this figure is available in the online journal.)

SN 2006mr (Contreras et al. 2009; Folatelli et al. 2009). $\Delta m_{15}(B)=1.82 \pm 0.02$. The near-IR maxima occurred 3-4 days after $T\left(B_{\max }\right)$. Its host was NGC 1316 (Fornax A), whose distance modulus is $m-M=31.59 \pm 0.08 \mathrm{mag}$ (Cantiello et al. 2007). Using the zero reddening line of Lira (1995) implies that this object has negative reddening, so we shall adopt $E(B-V)_{\mathrm{Gal}}=0.021$ (Schlegel et al. 1998) as the color excess. The implied extinctions in the $J$ and $H$ bands are then 0.018 and $0.012 \mathrm{mag}$, respectively. The Las Campanas photometry gives $J_{\max }=13.99 \pm 0.03$ and $H_{\max }=13.85 \pm 0.04$. The resulting absolute magnitudes are $M_{J}=-17.62 \pm 0.10$ and $M_{H}=-17.75 \pm 0.10$.

We do not consider the fast decliner SN 2000bk, which had a decline rate of $\Delta m_{15}(B)=1.63$ (Krisciunas et al. 2001). The first optical photometry was only obtained 10.8 days after the derived time of $B$-band maximum. The first near-IR data were obtained at $t=+5.9$ days. We do not know if it peaked early or late in the near-IR compared to $B$. Also, the $H$-band data were somewhat ragged.

Also, we do not consider the unusual SN 2005hk (Phillips et al. 2007). Along with SN 2002cx (Li et al. 2003), it may belong to a new subclass of Type Ia SNe. In their paper on the very subluminous SN 2008ha, Foley et al. (2009) list 14 objects similar to SN 2002cx. Valenti et al. (2009) even suggest that these $\mathrm{SNe}$ are core collapse objects, not Type Ia SNe.

In Figure 8 , we show the $J$-band photometry of six fast declining Type Ia SNe out to $t=35$ days. The top four objects are consistent with the stretchable template of Krisciunas et al. (2004b). They peak prior to $T\left(B_{\max }\right)$. SNe $2005 \mathrm{ke}$ and $2006 \mathrm{mr}$, on the other hand, cannot be fitted with same template no matter how it is stretched. That is because these objects peaked in the $J$-band after $T\left(B_{\max }\right)$. Note also that the late peakers have much

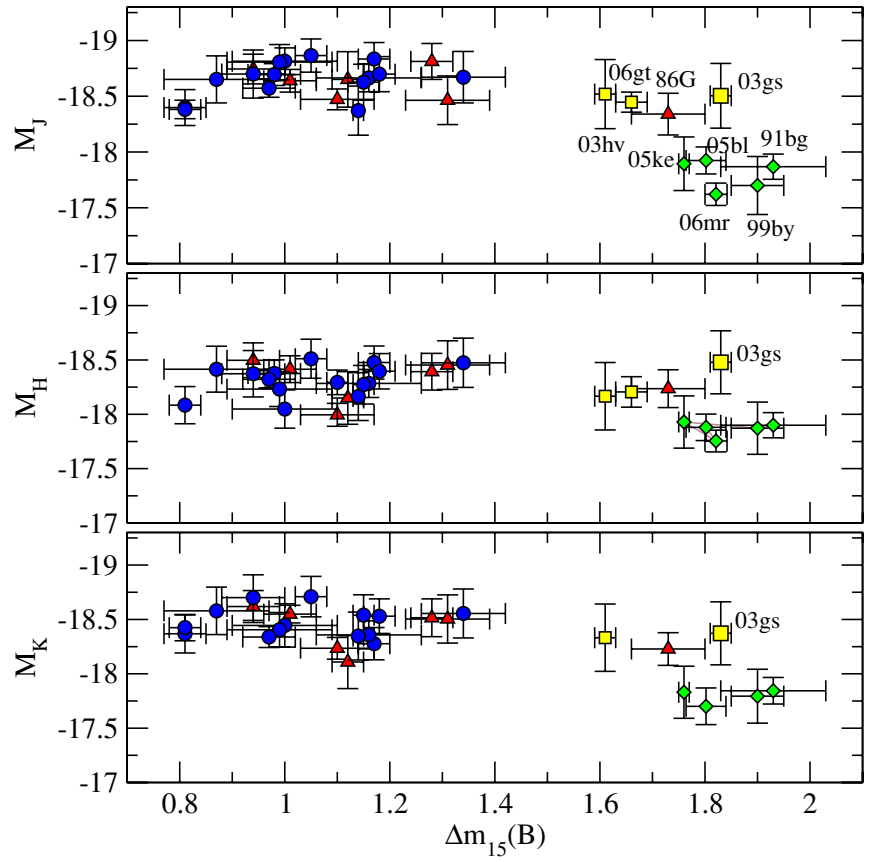

Figure 9. Near-IR decline rate relations for Type Ia SNe (absolute magnitudes at peak brightness vs. $\left.\Delta m_{15}(B)\right)$. Blue dots represent objects from Krisciunas et al. (2004c) which are in the smooth Hubble flow, with radial velocities in the CMB frame greater than $3000 \mathrm{~km} \mathrm{~s}^{-1}$. Red triangles represent objects from Krisciunas et al. (2004c) which are not far enough to be in the smooth Hubble flow; their distances were determined via Cepheids or SBF distances. SNe 2005bl and $2006 \mathrm{gt}$ are the only fast decliners that have Hubble flow distances. The objects that peak $\sim 3$ days before $B$-band maximum constitute a brighter subsample in each subdiagram. The objects that peak a few to several days after $B$-band maximum are all faint by comparison.

(A color version of this figure is available in the online journal.)

weaker secondary maxima. These are two of five known fast decliners that have fainter IR absolute magnitudes at maximum.

\subsection{A Bimodal Distribution of Absolute Magnitudes at Maximum}

In Figure 9, we show the near-IR absolute magnitudes from Figure 16 of Krisciunas et al. (2004c), along with SN 2000cf (Krisciunas et al. 2006), SN 2004S (Krisciunas et al. 2007), SN 2003gs, and the fast decliners mentioned above. We have made the values of SN $1980 \mathrm{~N}$ brighter by $0.15 \mathrm{mag}$, as we previously had adopted a distance modulus of 31.44 mag for its host, which happens to be the same host as that of SN 2006mr.

At first glance, Figure 9 indicates that there is just some range of near-IR absolute magnitudes of Type Ia SNe at maximum light. But taking the data at face value, we know of four fast declining Type Ia SNe (1986G, 2003gs, 2003hv, and 2006gt) that peaked early in the near-IR and whose near-IR absolute magnitudes at maximum light were not faint. We may also consider five fast declining Type Ia SNe that peaked late in the near-IR and whose near-IR absolute magnitudes at maximum light were on average significantly fainter than the mean values for Type Ia $\mathrm{SNe}$ that peak $\sim 2-3$ days before $T\left(B_{\max }\right)$. Those late peaking fast decliners are, on average, $0.81 \mathrm{mag}$ fainter in $M_{J}$, $0.44 \mathrm{mag}$ fainter in $M_{H}$, and $0.64 \mathrm{mag}$ fainter in $M_{K}$ than the early peakers. We suggest that there is a bimodal distribution of maximum-light near-IR absolute magnitudes of Type Ia SNe. Which group a particular object falls into depends on whether it peaks early or late compared to $T\left(B_{\max }\right)$. A summary of the absolute magnitudes of the two groups is given in Table 8 . 


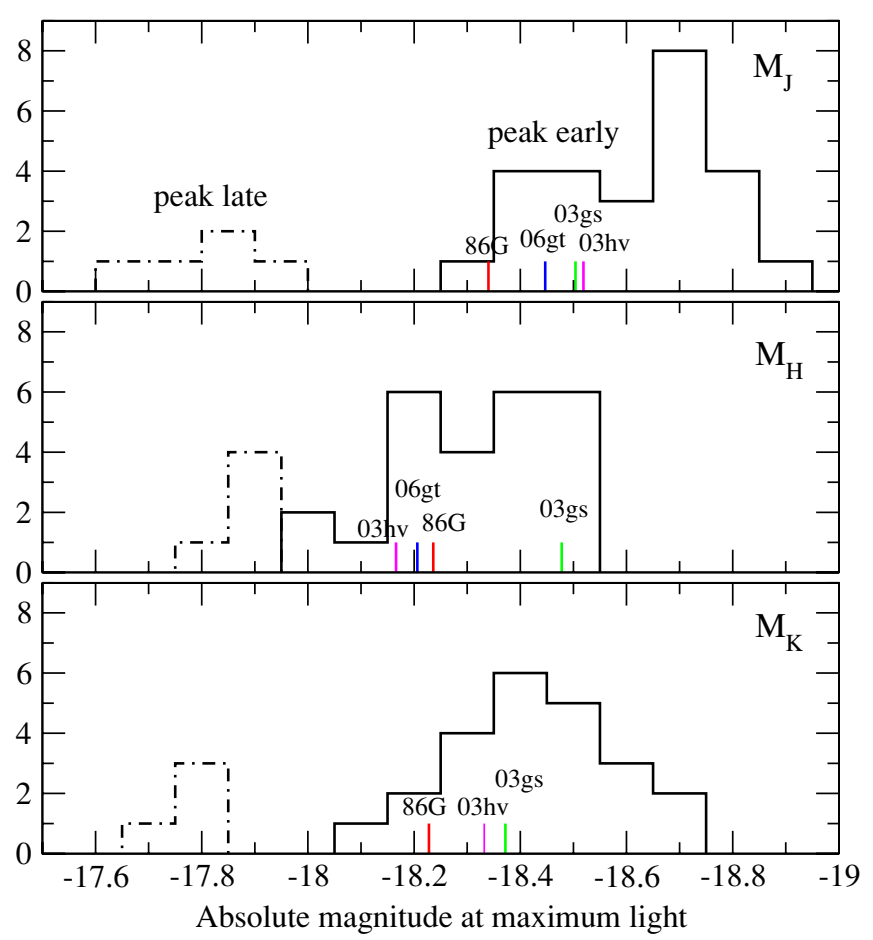

Figure 10. Histograms of the $J-, H$-, and $K$-band absolute magnitudes of Type Ia SNe plotted in Figure 9. We have grouped the objects according to the relative times of their infrared peaks compared to the time of $B$-band maximum. Those that peak early, including the fast decliners SNe 1986G, 2003gs, and 2006gt are brighter at maximum light than fast decliners that peak several days after $T\left(B_{\max }\right)$.

(A color version of this figure is available in the online journal.)

Table 8

Near-IR Absolute Magnitudes at Maximum ${ }^{\mathrm{a}}$

\begin{tabular}{lcccc}
\hline \hline Group & Filter & $\langle M\rangle$ & $\sigma_{x}$ & $N$ \\
\hline Peak early & & & & \\
& $J$ & -18.614 & \pm 0.158 & 25 \\
& $H$ & -18.308 & \pm 0.149 & 25 \\
Peak late & $K$ & -18.436 & \pm 0.153 & 23 \\
& $J$ & -17.802 & \pm 0.133 & 5 \\
& $H$ & -17.867 & \pm 0.067 & 5 \\
& $K$ & -17.792 & \pm 0.064 & 4 \\
\hline
\end{tabular}

Note.

a Type Ia SNe typically peak in the near-IR 3 days before $T\left(B_{\max }\right)$. Those that peak in the near-IR a few days after $T\left(B_{\max }\right)$ are fast decliners at optical wavelengths and are faint in all bands.

In Figure 10, we present histograms of the absolute magnitudes shown in Figure 9. For the $J$ - and $K$-band data, there is a noticeable bimodal distribution, depending on whether the objects peak early or late with respect to $T\left(B_{\max }\right)$. In the $H$ band, the two groups are not disconnected.

Another way of presenting the data for the fast decliners is shown in Figure 11, where we plot the $J$-band absolute magnitudes at maximum light versus the difference in the times of maximum light in the $J$ band and the $B$ band. A regression line is fitted to seven of the nine points. For SN 1991bg, on the basis of scanty data, $T\left(J_{\max }\right)$ occurred 2.1 days or less after $T\left(B_{\max }\right)$. For SN 1999 by, we assume that the $J$-band maximum occurred at the same time as the I-band maximum, which was well covered (Garnavich et al. 2004).

An easy way to understand the "early and bright" versus "late and faint" situation is as follows. For a (brighter) SN which has

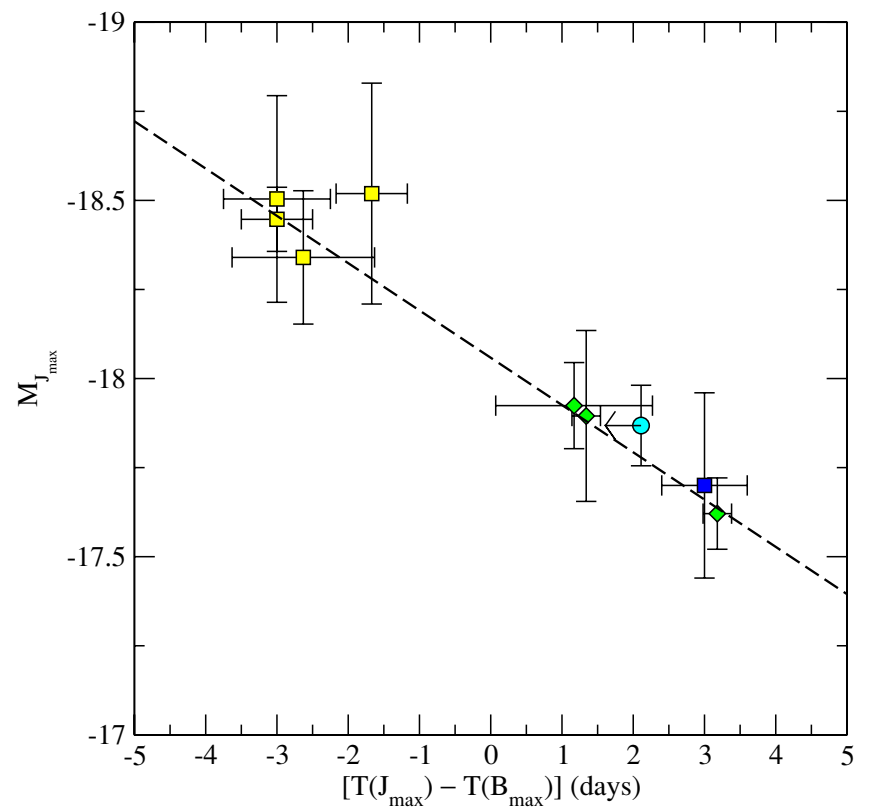

Figure 11. $J$-band absolute magnitudes at maximum light for nine fast declining Type Ia SNe vs. the relative time of the $J$-band and $B$-band maxima. The dashed line is a fit to the points representing SNe 2003gs, 2006gt, 1986G, and 2003hv (upper left), 2005bl, 2005ke, and 2006 mr (green diamonds, lower right). The cyan circle is for SN 1991bg and is an upper limit in the time domain. The blue square is for SN 1999by and assumes that the time of $J$-band maximum occurred at the same time as the $I$-band maximum.

(A color version of this figure is available in the online journal.)

two distinct humps in the light curve, these two humps occur at times $t_{1}$ and $t_{2}$. As long as there are two humps, $t_{\max }=t_{1}$. As we proceed to weaker explosions, $\left(t_{2}-t_{1}\right)$ decreases. Finally, when $t_{2}$ occurs soon after $t_{1}$, the two humps merge. In this case what we observe is a single hump whose maximum occurs at

$$
t_{\max }^{\prime}=\frac{\alpha t_{1}+t_{2}}{1+\alpha},
$$

where $\alpha \gtrsim 2$ for the $I$ - and $J$-band light curves. Details on modeling the secondary maxima are discussed by Höflich et al. (1995, Section 3) and Kasen (2006).

All of the slow decliners and mid-range decliners that have been observed early peaked early. Only the fast decliners that peak late appear to be fainter in the near-IR. We make two predictions: (1) Type Ia SNe that peak early are standard candles in the near-IR at peak brightness and (2) Type Ia SNe that peak a few to several days after $T\left(B_{\max }\right)$ in the near-IR are subluminous in all bands. We would, of course, be interested in the photometric and spectroscopic properties of any slow decliner or mid-range decliner that peaks several days after $T\left(B_{\max }\right)$. So far no such object has been observed.

On the basis of the data for nine fast declining Type Ia SNe, we assert that the absolute magnitudes at maximum light in the near-IR are related to the relative times of the near-IR maxima and $T\left(B_{\max }\right)$. A considerably larger data set is required to demonstrate for certain if it is a bimodal distribution or a linear trend.

\subsection{Bolometric Light Curve and the Mass of ${ }^{56} \mathrm{Ni}$}

Using the UBVRIJHK fluxes, the value of the distance modulus listed in Table 1, and extinction given in Section 2, we constructed an optical-near-infrared (OIR) pseudo-bolometric 


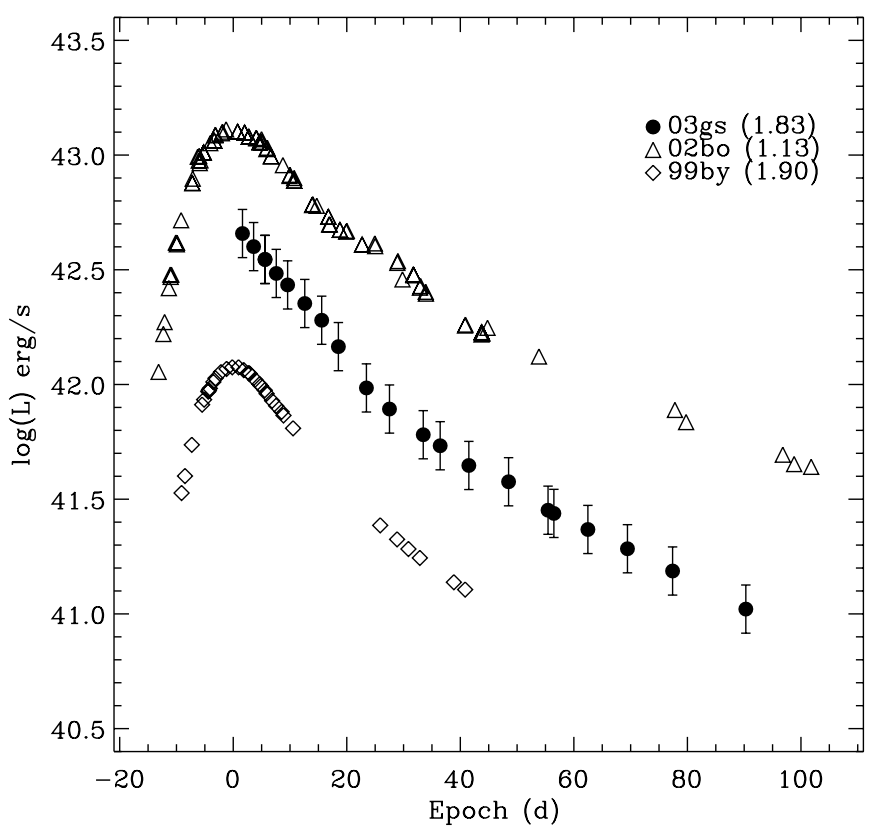

Figure 12. Quasi-bolometric light curves for SN 2003gs, SN 2002bo, and $\mathrm{SN} 1999 \mathrm{by} ; \Delta m_{15}(B)$ for each $\mathrm{SN}$ is indicated in parentheses. The error bars include uncertainties in distance and reddening, and are shown for SN $2003 \mathrm{gs}$ only.

Table 9

${ }^{56} \mathrm{Ni}$ Masses Estimated Using Arnett's Rule

\begin{tabular}{ccc}
\hline \hline SN & $\Delta m_{15}(B)$ & $M\left({ }^{56} \mathrm{Ni}\right) / M_{\odot}$ \\
\hline $2003 \mathrm{gs}$ & 1.83 & 0.25 \\
$1999 \mathrm{by}$ & 1.90 & 0.09 \\
$2005 \mathrm{bl}$ & 1.93 & 0.10 \\
$1991 \mathrm{bg}$ & 1.94 & 0.07 \\
$1998 \mathrm{de}$ & 1.95 & 0.06 \\
\hline
\end{tabular}

Note.

a Values of $\Delta m_{15}(B)$ for all SNe other than SN 2003gs are taken from Taubenberger et al. (2008, and references therein).

light curve, shown in Figure 12. We included a correction for the UV flux as described by Suntzeff (1996).

The peak of the bolometric light curve is directly related to the amount of ${ }^{56} \mathrm{Ni}$ produced in the explosion. It is well known that the peak quasi-bolometric luminosities of Type Ia SNe span a range of at least a factor of 25, implying a fairly large range of ${ }^{56} \mathrm{Ni}$ masses. Although our observations of SN $2003 \mathrm{gs}$ began after maximum light, we can still estimate the amount of ${ }^{56} \mathrm{Ni}$, bearing this caveat in mind. To do so, we employ Arnett's rule (Arnett 1982) as parameterized by Stritzinger \& Leibundgut (2005, their Equation (7)), and obtain a ${ }^{56} \mathrm{Ni}$ mass of about $0.25 M_{\odot}$. Stritzinger \& Leibundgut (2005) assumed a bolometric rise time for normal Type Ia SNe $\left(\Delta m_{15}(B) \sim 1.1\right)$ to be 19 days. This value may not be quite appropriate for objects like SN 2003gs, which have fast-evolving light curves.

Recently, Taubenberger et al. (2008, their Figure 6) collected the available photometry for a number of Type Ia SNe having $1.88 \lesssim \Delta m_{15}(B) \lesssim 1.95$ and compiled quasi-bolometric light curves for these. For the fast decliners in their sample, the peak luminosities ranged from $\sim 10^{42.05}-10^{42.3} \mathrm{erg} \mathrm{s}^{-1}$. We report the resulting values of the ${ }^{56} \mathrm{Ni}$ mass as obtained using the parameterization described above in Table 9, and find that all of them cluster in the $0.1 M_{\odot}$ range.

Interestingly, the narrow and rapidly declining light curve of SN $2003 \mathrm{gs}$ as indicated by its $\Delta m_{15}(B)$ of 1.83 belies the lower

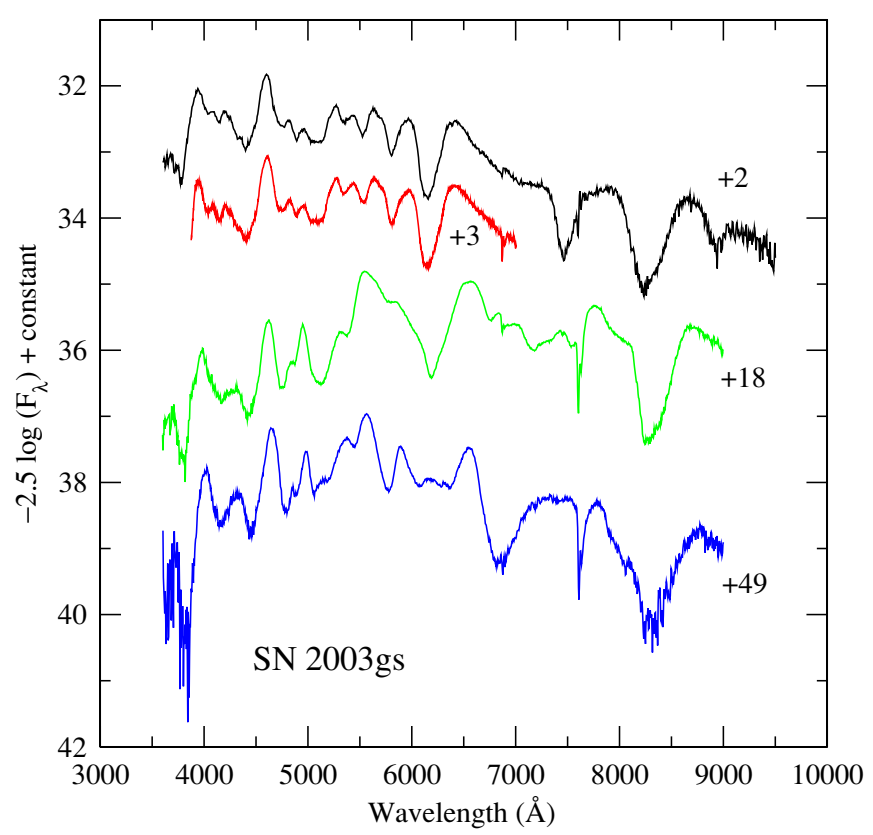

Figure 13. Four optical spectra of SN 2003gs. The numbers of days since $T\left(B_{\max }\right)$ are given in the plot.

(A color version of this figure is available in the online journal.)

limit to its peak luminosity ( $~ 10^{42.7} \mathrm{erg} \mathrm{s}^{-1}$ ). This may be taken to be further evidence that Type Ia SNe do not form a one-parameter family.

\subsection{Spectroscopy}

Optical spectra of SN 2003gs (Suntzeff et al. 2003; Matheson \& Suntzeff 2003; Hamuy et al. 2003) obtained with the CTIO $1.5 \mathrm{~m}$ telescope and also the Magellan telescopes soon after $T\left(B_{\max }\right)$ show the presence of Ti II in the region $4000-4500 \AA$ and a large ratio of $\mathrm{Si}$ II at $5800 \AA$ to $\mathrm{Si}$ II at $6150 \AA$ that are characteristics of moderately fast declining Type Ia $\mathrm{SNe}$ as described in Garnavich et al. (2004).

These spectra are shown in Figure 13. Using the Supernova Identification code SNID (Blondin \& Tonry 2007), we find that the two spectra obtained near maximum light are very similar to those of SN 2004eo (Pastorello et al. 2007b) at a comparable epoch. SN 2004eo had $\Delta m_{15}(B)=1.46$ and produced $0.45 M_{\odot}$ of ${ }^{56} \mathrm{Ni}$, similar to $\mathrm{SN} 2003 \mathrm{hv}$. At $t=18$ and 49 days, however, our optical spectra of SN 2003gs are similar to the prototypical fast decliner SN 1991bg. Many more optical spectra of SN 2003gs are presented by R. Kotak et al. (2009, in preparation).

Near-IR $(0.8-2.5 \mu \mathrm{m})$ spectra provide a rich source of information about the physical characteristics of SNe Ia because many elements are undetectable or obscured by line blending at other wavelengths but produce strong lines in the near-IR (Meikle et al. 1996; Wheeler et al. 1998; Bowers et al. 1997; Höflich et al. 2002; Marion et al. 2003, 2009). Near-IR spectroscopic observations are particularly effective for characterizing the chemical structure of the SN at different layers because the optical depth for most lines is smaller in the near-IR than at shorter wavelengths so that a greater radial depth can be probed with each spectrum (Wheeler et al. 1998).

Frogel et al. (1987) present a complete near-IR spectrum of SN 1986G obtained at +12 days and three partial spectra through +24 days. The data of SN 1986G closely resemble the spectra of SN 2003gs obtained at similar epochs. R. Kotak 
et al. (2009, in preparation) present 11 near-IR spectra of SN 2003 gs obtained between +4 days to 91 days after $T\left(B_{\max }\right)$. These authors kindly loaned us their spectra for the calculation of near-IR $S$-corrections to the photometry. We will not give an analysis of their spectra here, except to note that strong lines of iron group elements are seen early in the sequence.

The strong and early presence of iron and cobalt in the spectra of SN $2003 \mathrm{gs}$ indicates a lower opacity in the covering layers for SN 2003gs than is found in normal Type Ia SNe. This could be due to asymmetries in the explosion that place the iron and cobalt line-forming regions physically closer to the surface of the $\mathrm{SN}$ or to an explosion that produced a larger quantity of ${ }^{56} \mathrm{Ni}$. It is also possible that the depth of the surrounding envelope is approximately the same, but the opacities at these wavelengths are reduced by some other mechanism. In Section 3.3, we showed that SN 2003gs has a larger ${ }^{56} \mathrm{Ni}$ mass than other fast declining Type Ia SNe (see Table 9). In any case, this is an important clue to understanding the photometric behavior of SN 2003gs, which indicates a faster than normal decline from approximately normal peak brightness. If the envelope surrounding the iron and cobalt line-forming regions is not as deep or is more transparent than in normal Type Ia SNe, then $\gamma$ rays that power the luminosity will escape more easily and the decline rate will increase.

\section{CONCLUSIONS}

We have presented one of the most complete photometric data sets available for a fast declining Type Ia SN. SN 2003gs was first observed on the date of $V$-band maximum light, some 2 days after $B$-band maximum. Our coverage continued without any serious gaps until $t=91$ days. We also obtained some late-time photometry.

We deduced that SNe 1986G, 2003gs, 2003hv, and 2006gt were fast declining objects that shared some interesting photometric characteristics. These objects were subluminous in the optical band passes, but their near-IR maximum-light absolute magnitudes were statistically equal to those of the slow decliners and mid-range decliners. Also, the near-IR maxima of these four objects apparently occurred prior to the time of $B$ band maximum light. In the case of SN 2003gs, we can say that the near-IR maxima did not occur "late," i.e., a few days after $T\left(B_{\max }\right)$. Type Ia SNe that had late near-IR maxima (SNe 1991bg, 1999by, 2005bl, 2005ke, and 2006mr) were subluminous at the times of the IR maxima. There appears to be a bimodal distribution of near-IR absolute magnitudes of Type Ia $\mathrm{SNe}$ at maximum light. Which group a particular object falls into depends on whether it peaked late or early. This empirical finding is undoubtedly related to the opacity in the expanding fireball, and should help us refine models of Type Ia SNe.

Near-IR spectral data appear to show that NIR opacities in the outer layers are lower in SNe 1986G and 2003gs than they are in normal Type Ia SNe. If the $\gamma$ rays are less confined, then the observed luminosity decline rate would be accelerated. That result is consistent with the photometric result for these objects that indicates a faster than normal decline from approximately normal peak brightness in the NIR.

We thank Rubina Kotak for access to IR spectra of SN 2003gs ahead of publication and for calculating the bolometric light curve of SN 2003gs. E.A.K. was supported by the REU program of the National Science Foundation. We particularly thank the Carnegie Supernova Project for access to data prior to publication. We thank Jose Luis Prieto for his BVRI light-curve fitting templates. We thank Peter Hoeflich for useful discussions relating to the "late and faint" effect. We made use of the NASA/IPAC Extragalactic Database (NED), data of the Two Micron All Sky Survey, and SIMBAD, operated at CDS, Strasbourg, France. Most of the optical and IR photometry was obtained with the CTIO $1.3 \mathrm{~m}$ telescope, which is operated by the SMARTS consortium. Without the rapid response made possible by SMARTS, this valuable data set would not have been obtained. M.H. and R.C. acknowledge support provided by FONDECYT through grant 1060808, the Millennium Center for Supernova Science through grant P06-045-F, Centro de Astrofísica FONDAP 15010003, Center of Excellence in Astrophysics and Associated Technologies (PFB 06). R.C. was supported by CONICYT through Programa Nacional de Becas de Postgrado grant D-2108082. Some of the infrared data in Table 5 were obtained as part of the European Supernova Collaboration (ESC).

\section{REFERENCES}

Ajhar, E. A., Tonry, J. L., Blakesless, J. P., Riess, A. G., \& Schmidt, B. P. 2001, ApJ, 559, 584

Altavilla, G., et al. 2007, A\&A, 475, 585

Arnett, W. D. 1982, ApJ, 253, 785

Benetti, S., et al. 2004a, MNRAS, 348, 261

Benetti, S., et al. 2004b, ApJ, 623, 1011

Bessell, M. S. 1990, PASP, 102, 1181

Blondin, S., \& Tonry, J. L. 2007, ApJ, 666, 1024

Bowers, E. J. C., et al. 1997, MNRAS, 290, 663

Branch, D., Baron, E., Thomas, R. C., Kasen, D., Li, W. D., \& Filippenko, A. V. 2004, PASP, 116, 903

Cantiello, M., et al. 2007, ApJ, 668, 130

Cardelli, J. A., Clayton, G. C., \& Mathis, J. S. 1989, ApJ, 345, 245

Contreras, C., et al. 2009, submitted

Elias-Rosa, N., et al. 2006, MNRAS, 369, 1880

Elias-Rosa, N., et al. 2008, MNRAS, 384, 107

Evans, R. 2003, IAU Circ., 8171

Filippenko, A., et al. 1992a, ApJ, 384, L15

Filippenko, A., et al. 1992b, AJ, 104, 1543

Folatelli, G., et al. 2009, submitted

Foley, R. J., et al. 2009, AJ, 138, 376

Freedman, W., et al. 2001, ApJ, 553, 47

Frogel, J. A., Gregory, B., Kawara, K., Laney, D., Phillips, M. M., Terndrup, D., Vrba, F., \& Whitford, A. E. 1987, ApJ, 315, L129

Garavini, G., et al. 2007, A\&A, 471, 527

Garnavich, P., et al. 2004, ApJ, 613, 1120

Hamuy, M., Gonzalez, L., \& Morrell, N. 2003, IAU Circ., 8173

Hamuy, M., Phillips, M. M., Maza, J., Suntzeff, N. B., Schommer, R. A., \& Avilés, R. 1995, AJ, 109, 1

Hamuy, M., Phillips, M. M., Suntzeff, N. B., Schommer, R. A., Maza, J., Smith, R. C., Lira, P., \& Avilés, R. 1996, AJ, 112, 2438

Hamuy, M., et al. 2006, PASP, 118, 2

Hamuy, M., et al. 2009, ApJ, in press

Hillenbrand, L. A., Foster, J. B., Persson, S. E., \& Matthews, K. 2002, PASP, 114,708

Höflich, P. A., Gerardy, C. L., Fesen, R. A., \& Sakai, S. 2002, ApJ, 568, 791

Höflich, P., Khokhlov, A. M., \& Wheeler, J. C. 1995, ApJ, 444, 831

Hough, J. H., Bailey, J. A., Rouse, M. F., \& Whittet, D. C. B. 1987, MNRAS, $227,1 \mathrm{P}$

Immler, S., et al. 2006, ApJ, 648, L119

Jensen, J. B., et al. 2003, ApJ, 583, 712

Jha, S., Branch, D., Cornock, R., Foley, R., Li, W. D., Swift, B., Casebeer, D., \& Filippenko, A. V. 2006, AJ, 132, 189

Kasen, D. 2006, ApJ, 649, 939

Kotak, R., et al. 2005, A\&A, 436, 1021

Krisciunas, K., Phillips, M. M., \& Suntzeff, N. B. 2004a, ApJ, 602, L81

Krisciunas, K., Prieto, J. L., Garnavich, P., Riley, J.-L. G., Rest, A., Stubbs, C., \& McMillan, R. 2006, AJ, 131, 1639

Krisciunas, K., et al. 2001, AJ, 122, 1616

Krisciunas, K., et al. 2003, AJ, 125, 166

Krisciunas, K., et al. 2004b, AJ, 127, 1664

Krisciunas, K., et al. 2004c, AJ, 128, 3034 
Krisciunas, K., et al. 2007, AJ, 131, 58

Landolt, A. U. 1992, AJ, 104, 340

Leibundgut, B. 1993, AJ, 105, 301

Leloudas, G., et al. 2009, A\&A, 505, 265

Li, W. D., et al. 2003, PASP, 115, 453

Lira, P. 1995, Master's thesis, Univ. Chile

Marion, G. H., Höflich, P., Gerardy, C. L., Vacca, W. D., Wheeler, J. C., \& Robinson, E. L. 2009, AJ, 138, 727

Marion, G. H., et al. 2003, ApJ, 591, 316

Matheson, T., \& Suntzeff, N. B. 2003, IAU Circ., 8172

Meikle, W. P. S. 2000, MNRAS, 314, 782

Meikle, W. P. S., et al. 1996, MNRAS, 281, 263

Mikolajewska, J., \& Szostek, A. 2003, IAU Circ., 8175

Milne, P. A., The, L.-S., \& Leising, M. D. 2001, ApJ, 559, 1019

Pastorello, A., et al. 2007a, MNRAS, 376, 1301

Pastorello, A., et al. 2007b, MNRAS, 377, 1531

Perlmutter, S., et al. 1999, ApJ, 517, 565

Persson, S. E., Murphy, D. C., Krzeminski, W., Roth, M., \& Rieke, M. J. 1998, AJ, 116, 2475

Phillips, M. M. 1993, ApJ, 413, L105

Phillips, M. M., et al. 1987, PASP, 99, 592

Phillips, M. M., et al. 2007, PASP, 119, 360
Pignata, G., et al. 2004, MNRAS, 355, 178

Pignata, G., et al. 2008, MNRAS, 388, 971

Prieto, J. L., Rest, A., \& Suntzeff, N. B. 2006, ApJ, 647, 501

Pskovskii, IU. P. 1977, SvA, 21, 675

Riess, A. G., et al. 1998, AJ, 116, 1009

Schlegel, D. J., Finkbeiner, D. P., \& Davis, M. 1998, ApJ, 500, 525

Stanishev, V., et al. 2007, A\&A, 469, 645

Stritzinger, M., \& Leibundgut, B. 2005, A\&A, 431, 423

Stritzinger, M., Leibundgut, B., Walch, S., \& Contardo, G. 2006, A\&A, 450, 241

Stritzinger, M., et al. 2002, AJ, 124, 2100

Suntzeff, N.B. 1996, in Proc. IAU Colloquium 145, Supernovae and Supernova Remnants, ed. R. McCray \& Z. Wang (Cambridge: Cambridge Univ. Press) 41

Suntzeff, N. B., Candia, P., \& Stritzinger, M. 2003, IAU Circ., 8171

Taubenberger, S., et al. 2008, MNRAS, 385, 75

Tonry, J. L., Dressler, A., Blakeslee, J. P., Ajhar, E. A., Fletcher, A. B., Luppino, G. A., Metzger, M. R., \& Moore, C. B. 2001, ApJ, 546, 681

Valenti, S., et al. 2009, Nature, 459, 674

Wheeler, J. C., Höflich, P., Harkness, R. P., \& Spyromilio, J. 1998, ApJ, 496, 908

Wood-Vasey, W. M., et al. 2008, ApJ, 689, 377 